\title{
Ginkgo biloba: A Treasure of Functional Phytochemicals with Multimedicinal Applications
}

\author{
Noor-E-Tabassum, ${ }^{1}$ Rajib Das $\left(\mathbb{D},{ }^{1}\right.$ Mashia Subha Lami, ${ }^{1}$ Arka Jyoti Chakraborty, \\ Saikat Mitra, ${ }^{1}$ Trina Ekawati Tallei $\mathbb{D}^{2,3}$ Rinaldi Idroes, ${ }^{4}$ \\ Amany Abdel-Rahman Mohamed $\mathbb{D}^{5}{ }^{5}$ Md. Jamal Hossain $\mathbb{D}^{6}{ }^{6}$ Kuldeep Dhama, ${ }^{7}$ \\ Gomaa Mostafa-Hedeab $\mathbb{D}^{8},{ }^{8}$ and Talha Bin Emran $\mathbb{D}^{9}$ \\ ${ }^{1}$ Department of Pharmacy, Faculty of Pharmacy, University of Dhaka, Dhaka 1000, Bangladesh \\ ${ }^{2}$ Department of Biology, Faculty of Mathematics and Natural Sciences, Sam Ratulangi University, Manado 95115, \\ North Sulawesi, Indonesia \\ ${ }^{3}$ The University Centre of Excellence for Biotechnology and Conservation of Wallacea, \\ Institute for Research and Community Services, Sam Ratulangi University, Manado 95115, North Sulawesi, Indonesia \\ ${ }^{4}$ Department of Pharmacy, Faculty of Mathematics and Natural Sciences, Universitas Syiah Kuala, Kopelma Darussalam, \\ Banda Aceh 23111, Indonesia \\ ${ }^{5}$ Department of Forensic Medicine and Toxicology, Zagazig University, Zagazig 44511, Egypt \\ ${ }^{6}$ Department of Pharmacy, State University of Bangladesh, 77 Satmasjid Road, Dhanmondi, Dhaka 1205, Bangladesh \\ ${ }^{7}$ Division of Pathology, ICAR-Indian Veterinary Research Institute, Izatnagar, Bareilly 243122, Uttar Pradesh, India \\ ${ }^{8}$ Pharmacology Department, Health Sciences Research Unit, Medical College, Jouf University, Sakaka, Saudi Arabia \\ ${ }^{9}$ Department of Pharmacy, BGC Trust University Bangladesh, Chittagong 4381, Bangladesh
}

Correspondence should be addressed to Gomaa Mostafa-Hedeab; gomaa@ju.edu.sa and Talha Bin Emran; talhabmb@bgctub.ac.bd

Received 10 December 2021; Accepted 31 January 2022; Published 28 February 2022

Academic Editor: Harquin Simplice Foyet

Copyright (c) 2022 Noor-E-Tabassum et al. This is an open access article distributed under the Creative Commons Attribution License, which permits unrestricted use, distribution, and reproduction in any medium, provided the original work is properly cited.

Ginkgo biloba is an ancient plant species that is thought to provide a variety of health benefits to living organisms and contains plenty of bioactive components, making it a chemically diversified plant. G. biloba has been shown to have a variety of medicinal and pharmacological properties, including anticancer, antidementia, antidiabetic, antiobesity, antilipidemic, antimicrobial, antioxidant, antilipid peroxidation, antiplatelet, anti-inflammatory, hepatoprotective, antidepressant, antiaging, immunomodulatory, antihypertensive, and neuroprotective effects and is frequently used to treat neurological, cardiovascular, and respiratory diseases, such as tardive dyskinesia. Therefore, this review described the therapeutic applications of G. biloba. In addition to describing the therapeutic potential, this review also evaluates the chemical constituents, toxicity, adverse effect, synergistic effect, and the clinical studies of this plant which have been utilized for therapeutic benefits but have demonstrated other consequences. The capacity of G. biloba components to act as free radical scavengers is critical, and combining its extract with other plant extracts has been shown to synergistically boost antioxidant properties. G. biloba used long-term or at high doses that resulted in some adverse effects. Severe drug interactions have also been reported in both animals and humans when combined with other medications. The available data established from both preclinical and clinical studies confirm the potential of G. biloba plant extract in various diseases. Besides, the safety and efficacy of $G$. biloba continue to require verification through additional experimentation to guide medicinal use. 


\section{Introduction}

Plant-based phytochemicals have been utilized for over 1000 years and serve as a major platform for novel drug discovery. Ginkgo biloba L. (family: Ginkgoaceae; English name: Maidenhair tree) is a key source of novel herbal medications containing many bioactive constituents with therapeutic efficacy. This plant species is ancient, deciduous, tall, and strong, with fan-shaped, irregularly lobed leaves, growing to heights of up to 40 meters [1]. The genus name "biloba" refers to the tree's two separate lobes, and the genus name Ginkgo is a phonetic translation of the tree's Japanese name. G. biloba is clearly classified within the plant kingdom, and this plant is often termed a "living fossil" because, evolutionarily, it is one of the oldest seed plants [2]. After the atomic bomb detonation in Hiroshima, Japan, in 1946, G. biloba was the first plant to germinate. Microorganisms, chemical pollutants, insects, and environmental factors have a little effect on species survival [2].

G. biloba has been used as a traditional medicinal plant for longer than 2000 years in China and other parts of the world [3] and is currently grown in Europe, Asia, Argentina, North America, and New Zealand [4]. Ample research investigations focusing on this plant have shown promising therapeutic benefits [5-10], such as the treatment of asthma, tuberculosis (TB), skin problems, stomach discomfort, bronchitis, hearing loss, nervousness, arteriosclerosis, thrombus formation, ischemic heart disease, and diabetes mellitus (DM) [5]. Combining G. biloba extract (GBE) with grape seed skin extracts, quercetin, green tea, resveratrol, and bilberry extracts has been shown to reduce diastolic blood pressure in hypertensive individuals [5]. In addition to antihypertensive effects, the GBE EGb 761 appears to display antioxidant properties [6,7]. The ability of EGb 761 to scavenge free radicals produced by reactive nitrogen species (RNS) and reactive oxygen species (ROS) is a critical contributor to the medicinal effects of this extract $[8,9]$. When EGb 761 is combined with other plant extracts, the antioxidant capabilities are often synergistically enhanced. Promising chemical compounds that have been identified in this plant include flavonoids (quercetin, kaempferol, and isorhamnetin), terpenoids (bilobalide and ginkgolides), bioflavonoids (ginkgetin, sciadopitysin, and isoginkgetin), and organic acids (ginkgolic acid), which can affect a variety of biological processes [11]. As a result, G. biloba leaf extract is widely utilized to treat cardiovascular and neurological disorders $[3,12]$ and is one of the most commonly used traditional medicinal plants. Best health supplements available in the market that contain G. biloba are Nutricost Ginkgo biloba, GreeNatr Panax Ginseng G. biloba tablets, Nature's Bounty G. biloba, and VH Nutrition G. biloba $[13,14]$.

In recent years, clinical studies examining the efficacy and safety of herbal medicines and investigations into their underlying pharmacological functions have received a lot of attention. However, studies profiling the biopharmaceutical characteristics of herbal medicines, including efficacy and safety, are less common [15-19]. Plant-based medical products are expected to meet the same potency, purity, and biological content standards for efficacy that have been established for chemically defined synthetic medicines and dietary supplements, which are often not satisfied [15].

The fundamental aim of this review is to discuss the dissemination of $G$. biloba, including trade patterns, and provide an overview of the bioactive compounds found in this plant, including therapeutic effects, the molecular pathways thought to mediate these effects, nutritional values, the potential for toxicity, and interactions with other medications and food supplements.

\section{Chemical Constituents}

Several chemical compounds have been derived from $G$. biloba with a wide range of therapeutic activities. In recent years, novel chemical compounds, including new terpenoids and lignans, have been identified in G. biloba [20], as described in the following sections.

2.1. Flavonoids. Liquid chromatography-mass spectrometry (LC-MS) has been used to identify and separate several flavonoids found in G. biloba [21-25]. To date, 110 flavonoids have been identified, including kaempferol 3-O- $\alpha-1-$ [6000-p-coumaroyl $(\beta$-d)-glucopyranosyl $(1,2)$-rhamnopyranoside]-7-O- $\beta$-d-glucopyranoside and isorhamnetin 3-O$\alpha$-1-[6000-p-coumaroyl $(\alpha$-d)-glucopyranosyl $(1,2)$-rhamnopyranoside], which were identified in an $\mathrm{n}-\mathrm{BuOH}$ extract of G. biloba leaves. These flavonoids exhibit antioxidant properties when they bind with six other flavonol glycosides: quercetin 3-O- $\beta$-D-glucopyranoside, quercetin 3-O- $\beta$-rutinoside, quercetin 3-O- $\alpha$-L- $\left[6^{\prime \prime \prime}-\mathrm{p}\right.$-coumaroyl- $(\beta-\mathrm{D})$-glucopyranosyl-(1,2)-rhamnopyranoside], kaempferol 3-O- $\alpha$-L[6"' -p-coumaroyl- $(\beta-D)$-glucopyranosyl- $(1,2)$-rhamnopyranoside], quercetin 3-O- $\beta$-D-glucopyranosyl-(1-2)-alpha-Lrhamnopyranoside, and quercetin 3-O- $\alpha$-L- $\left[6^{\prime \prime \prime}-\mathrm{p}\right.$-coumaroyl- $(\beta-\mathrm{D})$-glucopyranosyl-(1,2)-rhamnopyranoside]-7O- $\beta$-D-glucopyranoside [26]. Figure 1 shows the flavonol structures and other flavonoids. Flavonoids can be characterized into seven groups: flavanones, isoflavones, flavone, biflavones, flavan-3-ols, flavonols, and biginkgosides. Ma et al. first separated and described biginkgosides in 2016, describing the isolation of nine biginkgosides [27].

2.2. Terpenoids. Ten diterpenoid lactones have been discovered, known as ginkgolides $\mathrm{Q}, \mathrm{P}, \mathrm{N}, \mathrm{M}, \mathrm{L}, \mathrm{K}, \mathrm{J}, \mathrm{C}, \mathrm{B}$, and A. Until recently, bilobalide was thought to be the only sesquiterpene lactone in G. biloba, but Dong et al. announced a new bilobalide isomer in 2020 [28]. G. biloba also contains nor-terpenoids, including three nor-sesquiterpenoids discovered by Shu et al. in G. biloba L. [29]. The chemical structures of terpenoids are shown in Figure 1.

2.3. Alkylphenols and Alkylphenolic Acids. Alkylphenols can be divided into five groups: cardols, cardanols, $\alpha$-hydroxycardanols, urushiols, isourushiols, and alkylphenolic acids. These chemical constituents of G. biloba are shown in Figure 1 [30]. Although ginkgolic acids are known to be toxic 
<smiles>COc1ccc(-c2oc3cc(O)cc(O)c3c(=O)c2O)cc1</smiles><smiles></smiles>

Myricetin<smiles>COc1cc([C@H]2OC[C@@H]3[C@H](c4ccc(O[C@@H]5O[C@H](CO)[C@@H](O)[C@H](O)[C@H]5O)c(OC)c4)OC[C@H]23)ccc1O[C@@H]1O[C@H](CO)[C@@H](O)[C@H](O)[C@H]1O</smiles>

Pinoresinol diglucoside<smiles>O=c1cc(-c2ccc(O)cc2)oc2cc(O)cc(O)c12</smiles>

Apigenin<smiles>O=c1cc(-c2ccc(O)c(O)c2)oc2cc(O)cc(O)c12</smiles><smiles>O=c1cc(-c2ccccc2)oc2cc(O)c(O)c(O)c12</smiles><smiles>COc1cc(O)c2c(=O)cc(-c3ccc(O)cc3)oc2c1</smiles>

Baicalein<smiles>O=C1CC(c2ccc(O)c(O)c2)Oc2cc(O)cc(O)c21</smiles><smiles>OC[C@H]1O[C@@H](O)[C@@H](O)[C@H]1O</smiles>
Genkwanin

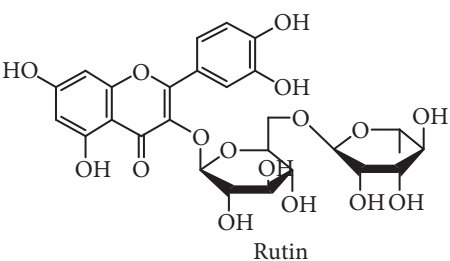

$\mathrm{OH} \mathrm{OH}$<smiles>O=c1cc(-c2ccc(O)cc2)oc2c(C3O[C@H](CO)[C@@H](O)[C@H](O)[C@H]3O)c(O)cc(O)c12</smiles><smiles>N#Cc1cc(O)cc2c1C(=O)C[C@@H](c1ccc(O)cc1)O2</smiles><smiles>Oc1cc(O)c2c(c1)O[C@H](O)[C@H](c1ccc(O)c(O)c1)C2</smiles><smiles>Oc1cc(O)c2c(c1)O[C@H](c1ccc(O)c(O)c1)[C@H](O)C2</smiles>

Epicatechin

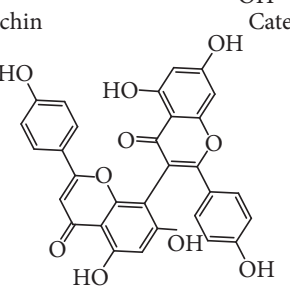

Amentoflavone

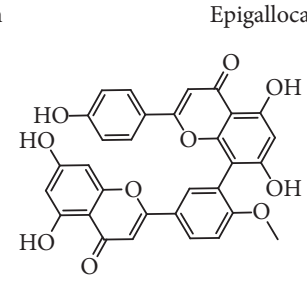

Bilobetin<smiles>O=c1c(-c2ccc(O)cc2)coc2cc(O)cc(O)c12</smiles>

Genistein<smiles>Oc1cc(O)c2c(c1)O[C@H](c1cc(O)c(O)c(O)c1)[C@H](O)C2</smiles>

Gallocatechin<smiles>COc1ccc(C2=CC(=O)C3=C(O)C=C(O)C(=C2O)C2=CC(c4ccc(OC)c(O)c4)=CC(=O)C2=C3)cc1</smiles>

Sciadopitysin<smiles>O=C(O)c1cc(O)c(O)c(OC(Cl)(Cl)Cl)c1</smiles><smiles>O=c1ccc2ccccc2o1</smiles>

Coumaric acid<smiles>O=C(O)/C=C\c1ccc(O)c(O)c1</smiles><smiles>COc1cc(/C=C/C(=O)O)ccc1O</smiles><smiles>COc1cc(CCCC(=O)O)cc(OC)c1O</smiles><smiles>C=CC=CCC=CCCCCCCc1cccc(O)c1</smiles>

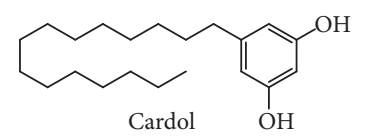

FIGURE 1: Chemical structures of flavonol, flavonoids (flavone, flavanone, isoflavone, flavan-3-ols, and biflavonoids), alkylphenols, alkylphenolic acids, carboxylic acids, and lignans.

[31], they have also been reported to display potential pharmacological effects.

2.4. Carboxylic Acids. The carboxylic acids that have been identified in G. biloba include ferulic acid, p-coumaric acid, protocatechuic acid, caffeic acid, p-hydroxybenzoic acid, m-hydroxybenzoic acid, vanillic acid, isovanillic acid, gallic acid, and sinapic acid [32], the structures of which are shown in Figure 1. Phenolic acids have been demonstrated to form glycosidic or covalent bonds, with the exception of the free form in GBL [20]. 
2.5. Lignans. Lignans were identified in G. biloba roots in 2015 and in G. biloba seeds in 2018 [33, 34]. Lignans obtained from $G$. biloba show antioxidant properties [33]. In 2018, lignans were also discovered in GBE [20]. Pinoresinol contains $0.012-0.020 \mathrm{mg} / \mathrm{mL}$ diglucoside and $1.05-1.87 \mathrm{mg} /$ $\mathrm{mL}$ total lignan glycosides. Five lignans were isolated from G. biloba by Shu et al. [29], as shown in Figure 1 .

2.6. Proanthocyanidins. Prodelphinidin and procyanidin are two proanthocyanidins that have been identified in G. biloba at a ratio of $85: 15$. Prodelphinidin is an epigallocatechin polymer, whereas procyanidin is comprised of epicatechin [20].

2.7. Polyprenols. Polyprenols, which are active ingredients identified in G. biloba, comprised of long chains of 14-24 isopentenyl units and have a similar structure as S-polyterpene alcohol (dolichols), which can be found in mammals, including people [35].

2.8. Polysaccharides. Okhti et al. examined the chemical structures of polysaccharides found in G. biloba and discovered that they were composed of glucose, rhamnose, mannose, arabinose, and galactose [36].

2.9. Others. The toxic component $4^{\prime}$-O-methylpyridoxine (MPN) was first discovered in G. biloba seeds and isolated by Klein et al. [37]. G. biloba essential oil contains 68 compounds, which includes $42.11 \%$ sesquiterpenes [38].

\section{Nutraceutical Value of Ginkgo biloba}

Nutraceuticals (also known as phytochemicals or functional foods) are bioactive chemical compounds found in nature that have beneficial properties, such as promoting health, preventing illness, or other medicinal effects. Nutraceuticals are used in a wide range of products produced by the pharmaceutical industry, the recently combined pharmaceutical/agribusiness industry, the food industry, and the herbal and dietary supplement sector [39]. G. biloba products were among the most widely sold medicinal products by US health food retailers, according to Blumenthal in 2000. Unlike ginkgo leaves, Ginkgo nuts have long been used as food and medicine and were first referenced in the herbals in approximately $1350 \mathrm{AD}$ [40]. G. biloba seeds contain high levels of vitamin C, carbohydrates, riboflavin, proteins, and various other nutrients. Ginkgo nuts are thought to offer health benefits, such as cancer prevention and neurologic disease treatment [41]. G. biloba seeds are an integral food crop and can be used in cakes, glazed vegetables, drinks, and alcohols, including various delicacies and sweets, depending on the cooking process used. Popular meals in China include Ginkgosteamed egg and Ginkgo-fried chicken, and Cheng Teng is a classic sweet treat found in Southeast Asia. G. biloba nuts have been served as a side dish in Japan since the Edo era (1600-1867) [42]. G. biloba seeds are also commonly used as conventional Chinese medical supplements for the prevention of fever, cough, and sputum production and to treat skin disorders, gonorrhea, toothache, and overactive bladder. G. biloba seeds are listed in the Chinese traditional medical reference Compendium of Materia Medica, and G. biloba seeds are commonly recommended as a functional food for the prevention of neurodegenerative diseases. However, the presence of ginkgolic and allergy-inducing acids, such as MPN and ginkgotoxin glucoids, can lead to vitamin B6 deficiencies and other unwanted side effects, preventing $G$. biloba products from becoming everyday foods [43]. Researchers have begun exploring methods for reducing levels of toxic substances found in G. biloba nuts using various processing methods. The incubation of G. biloba nuts in 3 volumes (v/w) of $5 \mathrm{~g} / \mathrm{L} \mathrm{Na}_{2} \mathrm{CO}_{3}$ at $15^{\circ} \mathrm{C}$ for $3 \mathrm{~h}$ was found to effectively remove ginkgolic acid from G. biloba nuts [44]. In addition, traditional food processing procedures, such as baking, boiling, and heating by microwaves, can also minimize seed toxicity. Because G. biloba seeds can be eaten as food and purchased freely in markets, the literature examining the bioactivities, manufacturing methods, and modified pharmacological functions of G. biloba seeds typically examine the use of seeds as food additives rather than as supplemental tablets or capsules (Table 1).

\section{Bioactive Compounds in Ginkgo biloba}

G. biloba leaves have been extensively investigated as a source of the plant's major medicinal components. Active chemical components found in G. biloba leaves include flavonoids and terpenoids, and plant extracts have exhibited a variety of pharmacological activities, including antibacterial, antioxidant, anti-inflammatory, antiallergic, and cytotoxic anticancer activities [4, 11]. Many other bioactive compounds, including bioflavonoids, organic acids, and polyprenols, have been identified in G. biloba (Table 2). Other constituents of $G$. biloba with known pharmacological activities are ginkgolides and bilobalide. Ginkgolides can be divided into five types ( $\mathrm{A}, \mathrm{B}, \mathrm{C}, \mathrm{J}$, and $\mathrm{M}$ ), in which each have a unique set of properties. Flavonoids, such as quercetin, kaempferol, and isorhamnetin, are found as glycoside derivatives in G. biloba (Figure 2). A standardized leaf extract of $G$. biloba, known as EGb 761, includes $6 \%$ terpenoids, $5 \%-24 \%$ flavonoid glycosides, $10 \%$ organic acids, and other bioactive compounds that are known to exert a wide range of beneficial health effects $[4,11]$.

\section{Pharmacological Activities}

G. biloba is a traditional medicinal herb with a variety of therapeutic properties. GBE is commonly used to treat asthma and bronchitis [59], and the leaves and nuts of this plant have been utilized as Chinese traditional medicine for millennia. G. biloba leaves have traditionally been used to treat heart issues and cure skin infections, whereas the nuts have traditionally been used to treat various types of respiratory illnesses, including asthma, chest pain, and cough, in addition to bladder irritation and alcoholism [60]. 
TABLE 1: Nutraceutical value of Ginkgo biloba [45].

\begin{tabular}{|c|c|}
\hline Main nutrition facts & Amount per $100 \mathrm{~g}$ \\
\hline Sodium & $7 \mathrm{mg}$ \\
\hline Potassium & $510 \mathrm{mg}$ \\
\hline Calcium & $2 \mathrm{mg}$ \\
\hline Saturated fatty acids & $0.319 \mathrm{~g}$ \\
\hline Calories & 182 Kcal (761.49 kj) \\
\hline Calories from fat & $14.0616 \mathrm{Kcal}(58.83 \mathrm{kj})$ \\
\hline Cholesterol & $0 \mathrm{mg}$ \\
\hline Food energy & Amount per $100 \mathrm{~g}$ \\
\hline Calories & 182 Kcal (761.49 kj) \\
\hline Calories from protein & $14.9904 \mathrm{Kcal}(62.82 \mathrm{kj})$ \\
\hline Calories from carbohydrate & $\begin{array}{c}153.032 \mathrm{Kcal} \\
(640.29 \mathrm{kj})\end{array}$ \\
\hline Calories from fat & $14.0616 \mathrm{Kcal}(58.83 \mathrm{kj})$ \\
\hline Fats and fatty acids & Amount per $100 \mathrm{~g}$ \\
\hline Total fat & $4.75 \pm 0.22 \mathrm{~g}$ \\
\hline Polyunsaturated fat & $0.618 \mathrm{~g}$ \\
\hline Monounsaturated fat & $0.619 \mathrm{~g}$ \\
\hline Saturated fat & $0.319 \mathrm{~g}$ \\
\hline Carbohydrates & Amount per $100 \mathrm{~g}$ \\
\hline Carbohydrate by difference & $72.98 \pm 0.20 \mathrm{~g}$ \\
\hline Protein and amino acids & Amount per $100 \mathrm{~g}$ \\
\hline Protein & $12.27 \pm 0.24 \mathrm{~g}$ \\
\hline Serine & $0.29 \mathrm{~g}$ \\
\hline Proline & $0.34 \mathrm{~g}$ \\
\hline Glycine & $0.23 \mathrm{~g}$ \\
\hline Leucine & $0.31 \mathrm{~g}$ \\
\hline Glutamic acid & $0.83 \mathrm{~g}$ \\
\hline Histidine & $0.1 \mathrm{~g}$ \\
\hline Valine & $0.28 \mathrm{~g}$ \\
\hline Phenylalanine & $0.17 \mathrm{~g}$ \\
\hline Arginine & $0.42 \mathrm{~g}$ \\
\hline Cystine & $0.02 \mathrm{~g}$ \\
\hline Alanine & $0.24 \mathrm{~g}$ \\
\hline Methionine & $0.05 \mathrm{~g}$ \\
\hline Aspartic acid & $0.54 \mathrm{~g}$ \\
\hline Lysine & $0.2 \mathrm{~g}$ \\
\hline Isoleucine & $0.2 \mathrm{~g}$ \\
\hline Threonine & $0.26 \mathrm{~g}$ \\
\hline Tryptophan & $0.07 \mathrm{~g}$ \\
\hline Tyrosine & $0.06 \mathrm{~g}$ \\
\hline Vitamins & Amount per $100 \mathrm{~g}$ \\
\hline Vitamin C & $15 \mathrm{mg}$ \\
\hline Vitamin B1 & $0.22 \mathrm{mg}$ \\
\hline Vitamin B2 & $0.09 \mathrm{mg}$ \\
\hline Vitamin B3 & $0.16 \mathrm{mg}$ \\
\hline Vitamin B6 & $0.328 \mathrm{mg}$ \\
\hline Total folate & $54 \mu \mathrm{g}$ \\
\hline Vitamin B9 & $0 \mu \mathrm{g}$ \\
\hline Folate (dietary folate equivalents) & $54 \mu \mathrm{g}$ \\
\hline Food folate & $54 \mu \mathrm{g}$ \\
\hline Vitamin B12 & $0 \mu \mathrm{g}$ \\
\hline Vitamin A & $558 \mathrm{iu}$ \\
\hline $\begin{array}{l}\text { Vitamin a (retinol activity } \\
\text { equivalents) }\end{array}$ & $28 \mu \mathrm{g}$ \\
\hline Retinol & $0 \mu \mathrm{g}$ \\
\hline Vitamin D & $0 \mathrm{iu}$ \\
\hline Vitamin D & $0 \mu \mathrm{g}$ \\
\hline Minerals & Amount per $100 \mathrm{~g}$ \\
\hline Manganese & $0.113 \mathrm{mg}$ \\
\hline Calcium & $2 \mathrm{mg}$ \\
\hline
\end{tabular}

TABle 1: Continued.

\begin{tabular}{lc}
\hline Main nutrition facts & Amount per $100 \mathrm{~g}$ \\
\hline Copper & $0.274 \mathrm{mg}$ \\
Iron & $1 \mathrm{mg}$ \\
Zinc & $0.34 \mathrm{mg}$ \\
Magnesium & $27 \mathrm{mg}$ \\
Sodium & $7 \mathrm{mg}$ \\
Phosphorus & $124 \mathrm{mg}$ \\
Potassium & $510 \mathrm{mg}$ \\
Sterols & Amount per $100 \mathrm{~g}$ \\
Cholesterol & $0 \mathrm{mg}$ \\
Other nutrients & Amount per $100 \mathrm{~g}$ \\
Ash & $10.01 \pm 0.06 \mathrm{~g}$ \\
Water & $55.2 \mathrm{~g}$ \\
\hline
\end{tabular}

G. biloba has been shown to effectively treat symptoms associated with Alzheimer's disease (AD), epilepsy, cerebrovascular illness, stroke, and peripheral vascular disease $[61,62]$. Evidence suggests that GBE exerts a variety of pharmacological activities, such as lowering the risk of cardiovascular disease, preventing ischemia-induced oxidation [63, 64], increasing cerebral blood flow [65], hepatoprotective functions [66, 67], and platelet antagonization [68]. GBEs have also been studied for anticancer effects, although this remains a poorly understood area. The whole extract derived from G. biloba fruit provides better effects for various indications than any specific isolated compounds [69].

5.1. Treatment of Respiratory Diseases. Acute respiratory distress syndrome (ARDS), asthma, and chronic obstructive pulmonary disease (COPD) are all associated with airway inflammation, and disease onset, progression, and prognosis are all considered to be influenced by inflammatory processes. Airway inflammation is exacerbated by neutrophils and other inflammatory cells. Under inflammatory conditions, neutrophils mediate two fundamental metabolic reactions: respiratory burst and chemotaxis. $\mathrm{Wu}$ et al. suggested that during lipopolysaccharide (LPS)-induced pulmonary injury, ginkgolide $\mathrm{M}(\mathrm{GM})$ and ginkgolide $\mathrm{B}$ (GB) were equally effective for reducing the aggregation of inflammatory cells, such as lymphocytes, neutrophils, and macrophages, and improving cytological lung damage [70]. According to Tao et al., the production of interleukin (IL)-4, IL-5, IL-6, IL-8, IL-13, and tumor necrosis factor (TNF)- $\alpha$ decreased considerably when allergy model mice were treated with EGb 761. EGb 761 also regulates the activity of leukocyte elastase, a protein involved in blood coagulation disorders, pulmonary damage, and chronic bronchitis, and the components of EGb 761 were able to reduce inflammation in the lungs [71].

5.2. Anticancer Effects. Cancer is a disease in which certain cells in the body begin to proliferate uncontrollably, eventually spreading to other areas of the body. Cancer is a global problem, and according to the National Institutes of Health, 
TABLE 2: Major bioactive components of Ginkgo biloba.

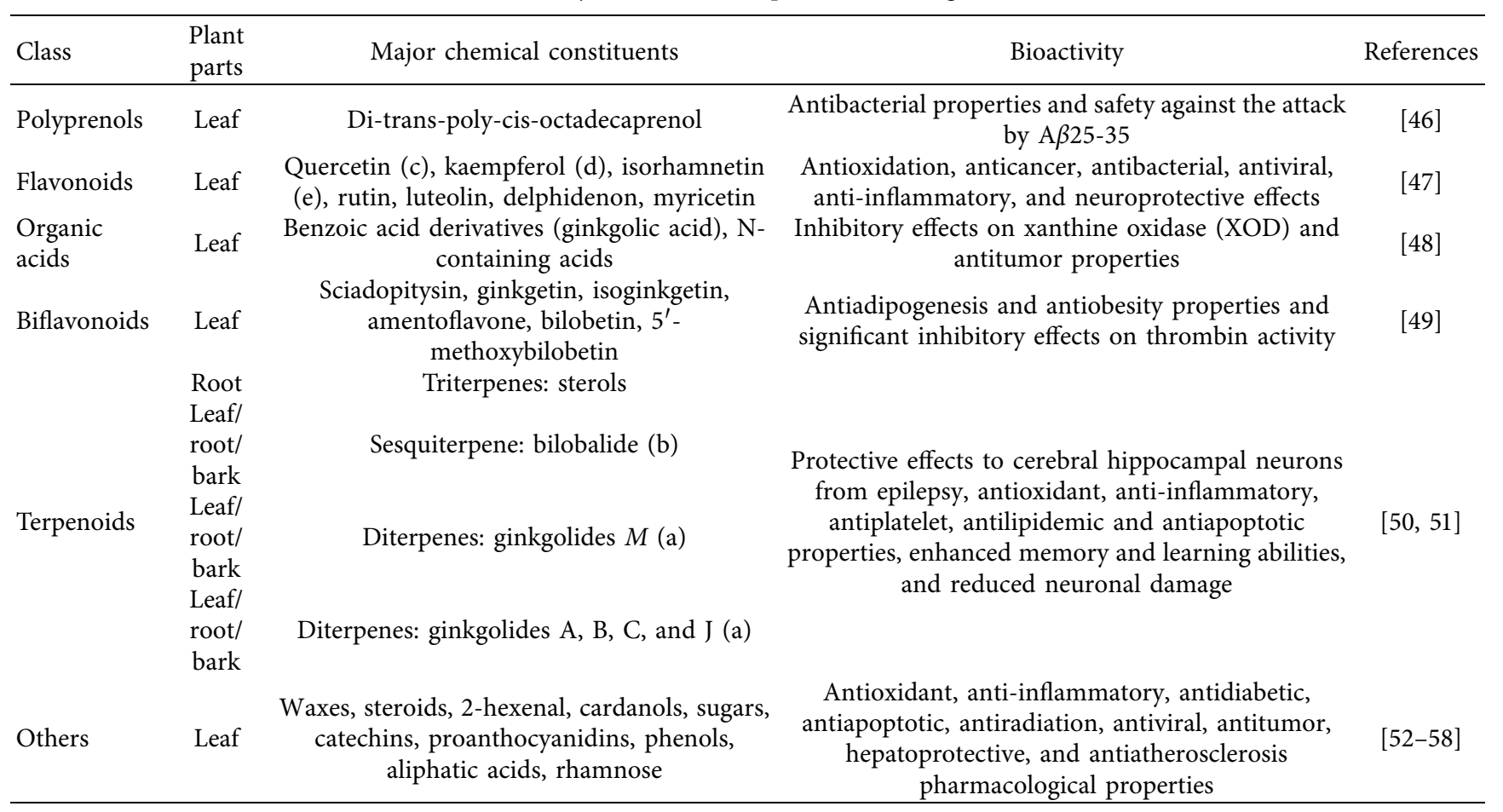<smiles>CC(C)(C)[C@@]1(O)C[C@H]2OC(=O)C[C@]23C(=O)O[C@H]2OC(=O)[C@H](O)[C@]213</smiles>

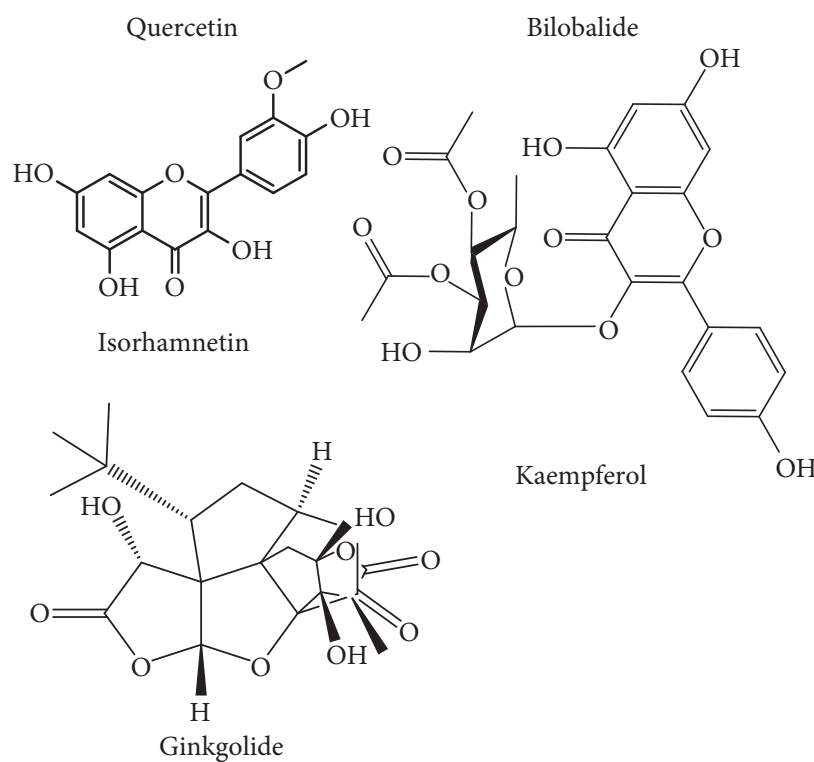

Figure 2: Chemical structures of Ginkgo biloba's key bioactive compounds. 
in 2016, 1.7 million new cancer patients were diagnosed with cancer in the United States alone, with 0.6 million people dying as a result of the disease, indicating the importance of developing novel cancer therapies. Ahmed et al. investigated the effectiveness of GBE for the treatment of rats with hepatocellular carcinoma (HCC). GBE significantly enhanced the histological characteristics of liver tissue, decreasing the levels of alpha-fetoprotein (AFP), glypican-3 (GPC-3), and carcinoembryonic antigen (CEA) in HCC rats via the increased gene expression of inhibitor of growth (ING)-3 and the decreased gene expression of the transcription factor forkhead box protein 1 (FOXP1) in the liver [72]. Another study conducted by Han et al. demonstrated the anti-Lewis lung cancer (LLC) efficacy of G. biloba exocarp extracts (GBEE), which limited in vitro LLC cell growth by regulating catenin and Wnt3a expression in a dose-dependent manner, and GBEE demonstrated similar effects on the expression of various proteins, including Wnt3a, vascular endothelial growth factor (VEGF), and phosphorylated (p)-protein kinase $\mathrm{B}$ (Akt)/Akt, when administered to C57BL/6J mice. GBEE decreased the protein expression levels of p-Akt/Akt, VEGF, VEGF receptor (VEGFR), and $\beta$-catenin, contributing to the inhibition of lung carcinoma metastasis. VEGF and VEGFR2 mRNA levels were similarly reduced, suggesting that GBEE suppresses tumor angiogenesis, resulting in antitumor effects. The underlying mechanisms of these outcomes were associated with the inhibition of the Wnt, catenin, and VEGF signaling pathways in LLC [73]. Qian et al. investigated the impacts of GBE on human SGC7901 gastric cancer cells in vitro and in vivo. GBE inhibited the development of SGC7901 gastric cancer cells in a dose and time-dependent manner, and GBE increased the number of cells in the G1 stage while decreasing the number of cells in the $\mathrm{S}$ stage, as assessed by flow cytometry. GBE-treated cells showed reduced mRNA and protein levels of cyclin D1 and $\mathrm{c}-\mathrm{Myc}$, indicating that GBE might inhibit cell cycle progression by reducing cyclin D1 and c-Myc production [74]. When Liu and colleagues used EGb 761 in stomach cancer cells, they observed similar effects; however, the underlying mechanisms of EGb 761 activity were restricted to the kinase suppressor of Ras 1 (KSR1)-mediated extracellular signalregulated kinase (ERK) 1/2 pathway [44].

5.3. Antidementia Effects. Most EGb 761 clinical trials are targeted at enhancing cognition and memory, with some concentrating on dementia, particularly dementia presentations associated with the gradual loss of cognition and memory, including vascular dementia, Lewy body dementia, and frontotemporal dementia. Because EGb 761 can prevent amyloid production and toxicity, control excitotoxic glutamatergic neurotransmission, and act as a radical scavenger, it could potentially be used to treat multiple dementia etiologies [75].

5.4. Antidiabetic Effects. DM is a complicated disease that is currently estimated to affect 366 million individuals globally, which is predicted to increase to 552 million affected individuals by 2030 [76]. Several in vivo investigations have demonstrated that GBE has antidiabetic effects. Bilobalide, according to one study, shielded lipid cells against oxygen deficiency-induced insulin resistance and reduced inflammation by increasing adiponectin secretion, blocking the serine phosphorylation of insulin receptor substrate 1 (IRS-1) receptors in the insulin signaling cascade, lowering inflammatory adipokine production, and decreasing nuclear factor kappa B (NF- $\kappa \mathrm{B}) / \mathrm{c}$-Jun N-terminal kinase (JNK) activation [77]. According to these findings, ginkgolide B (orally administered at $5 \mathrm{mg} / \mathrm{kg} /$ day) enhanced cholinergic vasorelaxation and phenylephrine vasoconstriction in male Sprague Dawley rats. Ginkgolide B also boosted superoxide dismutase (SOD) and endothelial nitric oxide (NO) synthase (eNOS) activity while decreasing $\mathrm{H}_{2} \mathrm{~S}$ production and malonaldehyde (MDA) levels. Ginkgolide B also improved the expression of cystathionine-lyase proteins, cystathioninesynthetase, NADP-oxidase subunits, and the glutathione peroxidase (GPX1) enzyme [35].

5.5. Antiobesity Effects. High-fat diets contribute to the pathogenesis of obesity by altering the regulation of the peripheral metabolism and food consumption at the level of the central nervous system (CNS), leading to weight gain, increased insulin tolerance, and other metabolic disorders [59]. GBE administration was found to be beneficial for reducing body weight. Food consumption over a 24-hour period was measured by assessing the difference between the quantity provided and quantity remaining. The gene expression levels of insulin receptor (IR), IL-10, and lipid receptor R1 (Adipo R1) and the phosphorylation of Akt all increased significantly, potentially stimulating the insulin signaling cascade. By contrast, TNF- $\alpha$ levels and NF- $\kappa$ B p65 phosphorylation were reduced. The anti-inflammatory activities of GBE, which reduces TNF- $\alpha$ levels in retroperitoneal fat deposits, can mitigate the negative effects associated with excessive high-fat diet consumption [78]. The likely mediators of these antiobesity effects are the GBE components ginkgetin, isoginkgetin, bilobetin, and sciadopitysin, which have moderate to strong inhibitory effects on pancreatic lipase (PL), with half-maximal inhibitory concentration $\left(\mathrm{IC}_{50}\right)$ values ranging from $2.90 \mathrm{M}$ to greater than 12.78 M, indicating that the compounds found in G. biloba may serve as lead compounds for the development of bioflavonoid-type PL inhibitors [79]. The impacts of ginkgolide $\mathrm{B}$ on hepatic steatosis and body weight were studied in a high-fat diet-induced model of obesity in C57BL/6 male mice, which revealed that treatment with ginkgolide $\mathrm{B}(0.1 \%$ per day) induced human pregnane $X$ receptor (hPXR) mRNA expression in the liver in a dose-dependent manner, without affecting the expression of peroxisome proliferatoractivated receptor (PPAR), liver $X$ receptor (LXR), farnesyl $X$ receptor (FXR), or LXR trans-activities. Ginkgolide B also reduced plasma triglyceride (TG) levels and body weights while resolving hepatic steatosis [80]. Treatment with 3-100 M ginkgolide C substantially decreased lipid aggregation in an in vitro study of HepG2 liver cells and increased TG degradation by increasing the phosphorylation of hormone-sensitive lipase and the production of adipose TG lipase. Ginkgolide C was found to enhance lipolysis and 


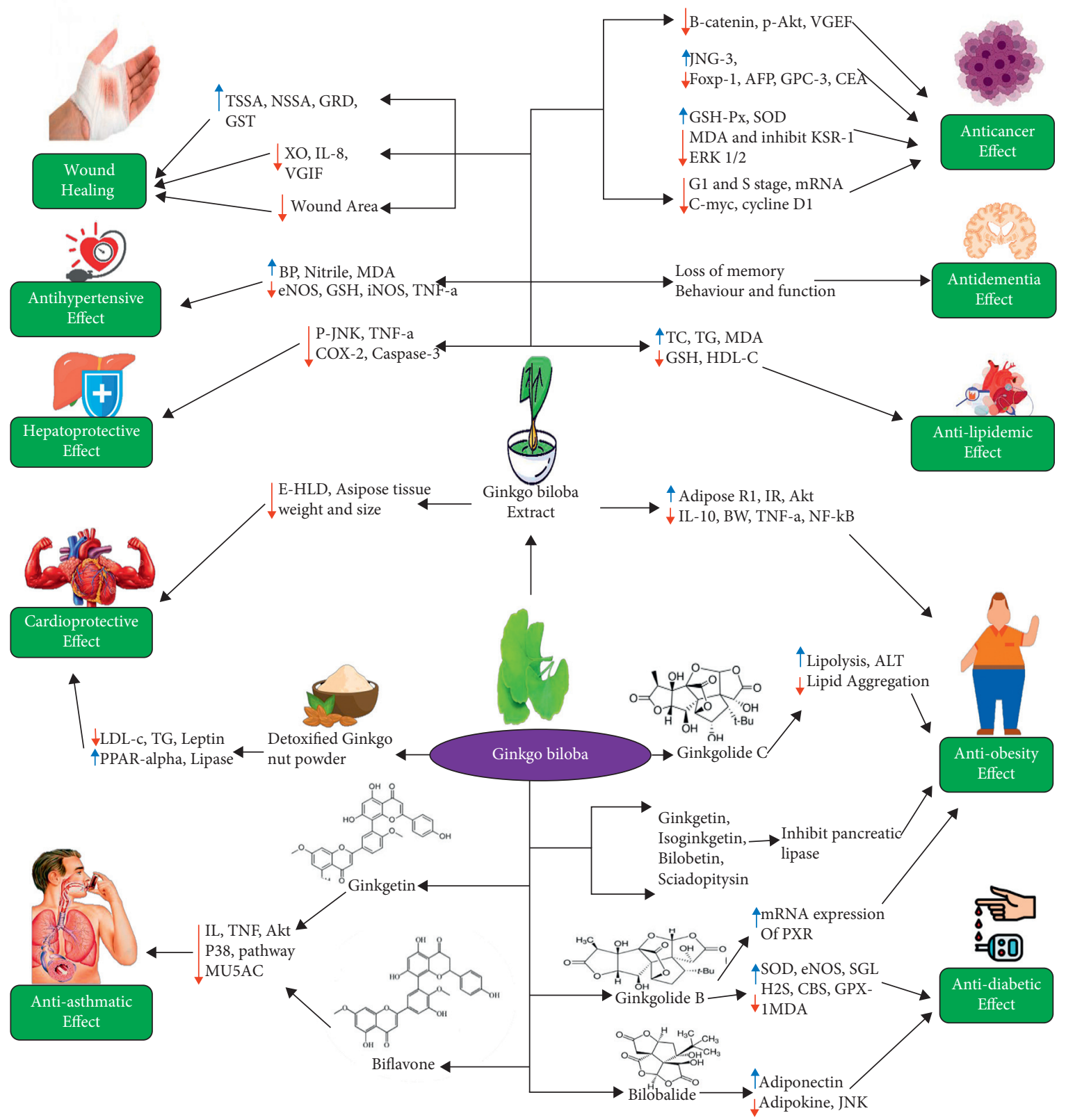

(a)

Figure 3: Continued. 


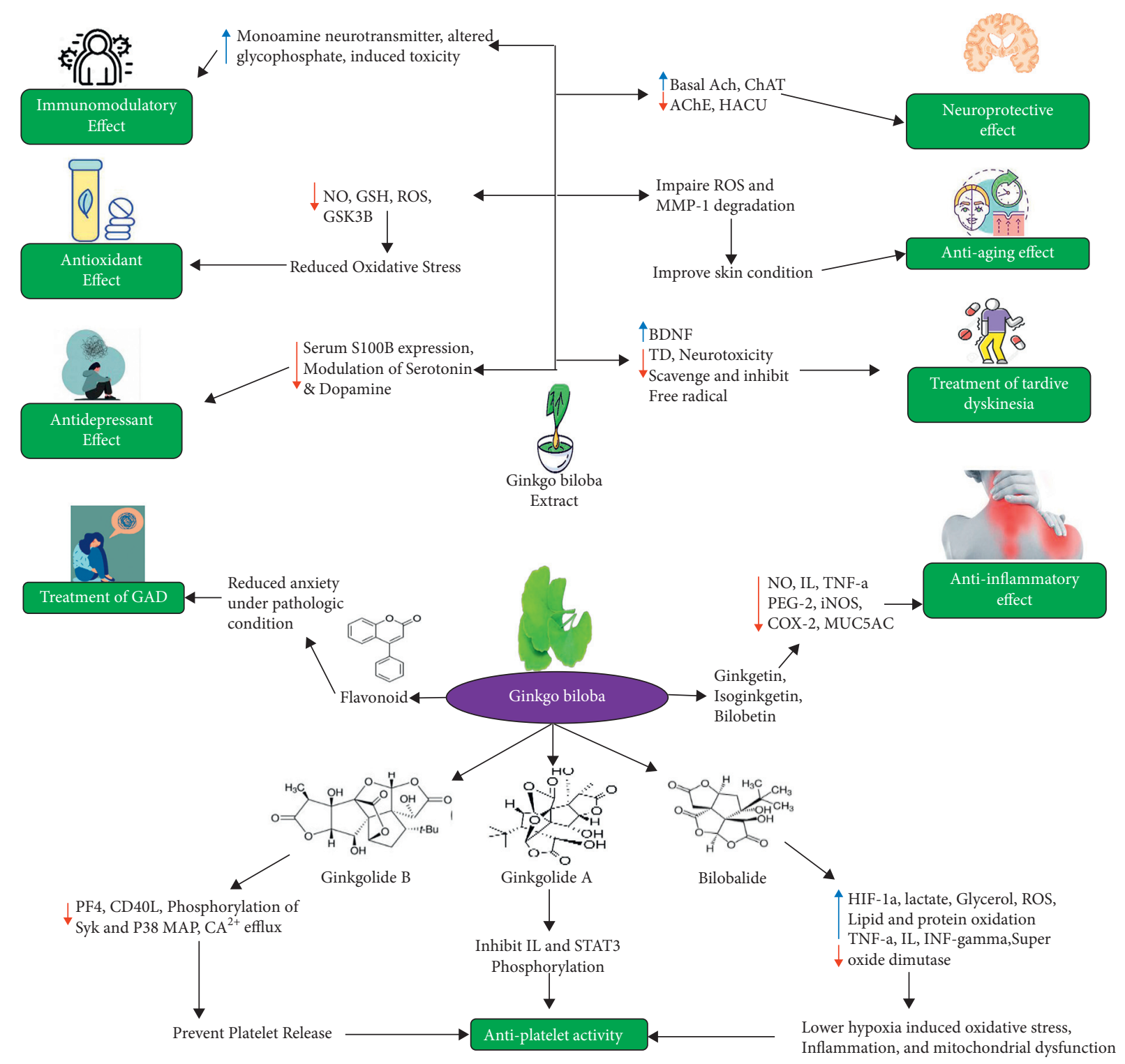

(b)

Figure 3: (a), (b) Therapeutic potential of Ginkgo biloba.

reduce lipid aggregation through the activation of the sirtuin 1 (SIRT1)-AMP-activated protein kinase (AMPK) cascade in an oleic acid-induced model of the fatty liver [80].

5.6. Antilipidemic Effect. Dyslipidemia is defined by high TG levels, low high-density lipoprotein cholesterol (HDL-C) levels, and increased low-density lipoprotein cholesterol (LDL-C) levels. In addition, hyperlipidemia has been associated with obesity and insulin resistance. In a separate study of male rabbits, G. biloba $(10 \mathrm{mg} / \mathrm{kg} / \mathrm{day})$ treatment dramatically decreased plasma cholesterol and TG levels while significantly increasing HDL-C levels compared with the control group. Furthermore, in aortic tissue, G. biloba has been found to lower MDA levels while raising glutathione (GSH) levels [81].
5.7. Treatment of Cardiovascular Diseases. The impacts of G. biloba seeds on the cholesterol metabolism were initially studied by Mahadevan et al., who studied the effects of the lipid metabolism and cardiovascular disease prevention mediated by various components of G. biloba seeds, including whole seeds, water-soluble fraction, and lipid-soluble fraction [60]. In vitro studies have shown that G. biloba seeds can impact the synthesis of apolipoprotein B and LDL receptor, modulating the blood cholesterol level of the liver. In vivo, whole seeds reduced hepatic cholesterol levels while increasing serum cholesterol levels. The lipid-soluble portion reduced hepatic cholesterol levels, whereas the water-soluble portion increased serum cholesterol levels, suggesting that lipid-soluble portion of $G$. biloba seeds could be used to lower the risks of heart disease. Ginkgo seed ethanol extract was shown to be beneficial in high-fat diet-fed mice. The 
TABLe 3: In vivo and in vitro studies on therapeutic effects of Ginkgo biloba.

\begin{tabular}{|c|c|c|c|c|c|c|}
\hline Field of study & $\begin{array}{c}\text { Bioactive } \\
\text { compounds }\end{array}$ & Subject & Dosage & Outcome & Mechanism of action & References \\
\hline \multirow{2}{*}{$\begin{array}{l}\text { Treatment of } \\
\text { respiratory diseases }\end{array}$} & $\begin{array}{l}\text { Ginkgetin, } \\
\text { biflavones }\end{array}$ & $\begin{array}{l}\text { In vivo, an } \\
\text { allergic mouse } \\
\text { model }\end{array}$ & $\begin{array}{c}0.01,0.1,1,10 \text {, and } \\
40 \mathrm{mg} / \mathrm{kg}\end{array}$ & $\begin{array}{l}\downarrow \text { IL-4, } \downarrow \text { IL-5, } \downarrow \text { IL-6, } \\
\downarrow \text { IL-8, } \downarrow \text { IL-13, } \downarrow \text { TNF }\end{array}$ & $\begin{array}{c}\text { Ginkgetin } \\
\text { significantly reduced } \\
\text { irregular expression of } \\
\text { the Akt and p38 } \\
\text { pathway, whereas } \\
\text { biflavones reduced } \\
\text { MUC5AC mRNA } \\
\text { expression }\end{array}$ & {$[71]$} \\
\hline & $\begin{array}{l}\text { Ginkgolides } \\
\text { mixture (GM) }\end{array}$ & $\begin{array}{l}\text { In vitro, RAW } \\
264.7 \text { cell } \\
\text { culture model }\end{array}$ & $\begin{array}{c}19.1,38.2 \text { or } \\
76.4 \mathrm{mg} / \mathrm{kg}\end{array}$ & $\begin{array}{c}\downarrow \text { LPS-induced IL-1 } \\
\text { mRNA and protein } \\
\text { levels, } \downarrow \text { IL-10 protein } \\
\text { level, } \downarrow \text { exudation of } \\
\text { plasma protein, } \\
\downarrow \text { myeloperoxidase } \\
\text { activities }\end{array}$ & $\begin{array}{l}\text { GB and GM were } \\
\text { shown to be a key } \\
\text { component in the } \\
\text { treatment of } \\
\text { lipopolysaccharide- } \\
\text { induced lung injury }\end{array}$ & {$[70]$} \\
\hline \multirow[t]{3}{*}{ Anticancer effects } & GBEE & $\begin{array}{c}\text { In vitro, } \\
\text { C57BL/6J mice }\end{array}$ & $\begin{array}{l}5,10,20,40,80 \\
\text { and } 160 \mu \mathrm{g} / \mathrm{mL}\end{array}$ & $\begin{array}{c}\downarrow \beta \text {-Catenin } \\
\text { expression, } \downarrow \text { p-Akt/ } \\
\text { Akt expression, } \\
\text { VEGF and VEGFR } \\
\text { expression, } \downarrow \text { mRNA } \\
\text { levels of VEGF and } \\
\text { VEGFR2 }\end{array}$ & $\begin{array}{l}\text { Treatment with } \\
\text { Ginkgo biloba leaf } \\
\text { extracts resulted in a } \\
\text { substantial decrease in } \\
\text { serum tumor markers } \\
\text { and oncogene } \\
\text { downregulation, as } \\
\text { well as upregulation of } \\
\text { a tumor suppressor } \\
\text { gene in liver tissue } \\
\text { GBEE demonstrated } \\
\text { the antitumor and } \\
\text { antimetastatic actions } \\
\text { due to the induction } \\
\text { of the Wnt/ } \beta \text {-catenin- } \\
\text { VEGF signaling } \\
\text { pathway, which } \\
\text { inhibited tumor } \\
\text { angiogenesis }\end{array}$ & {$[72]$} \\
\hline & GBE & $\begin{array}{l}\text { In vitro, gastric } \\
\text { carcinoma } \\
\text { SGC7901 cells }\end{array}$ & $\begin{array}{l}100,200,300 \\
400 \mathrm{mg} / \mathrm{L}\end{array}$ & $\begin{array}{c}\uparrow \text { G1 stage cell } \\
\text { percentage, } \downarrow S \text { stage } \\
\text { cell percentage, } \\
\downarrow \text { mRNA and protein } \\
\text { for the cyclin D1 and } \\
\text { c-myc genes }\end{array}$ & $\begin{array}{l}\text { GBE inhibited the } \\
\text { proliferation of gastric } \\
\text { carcinoma SGC7901 } \\
\text { cells }\end{array}$ & {$[74]$} \\
\hline & GBE & $\begin{array}{l}\text { In vitro, gastric } \\
\text { cancer cells }\end{array}$ & $\begin{array}{l}\text { GBE with } 0.9 \% \\
\mathrm{NaCl}\end{array}$ & $\begin{array}{c}\text { Suppressed and } \\
\text { inhibited expression } \\
\text { of KSR1, p-KSR1, } \\
\text { ERK1/2, and p- } \\
\text { ERK1/2, } \downarrow \text { MDA, } \\
\uparrow G S H-P x, \uparrow S O D\end{array}$ & $\begin{array}{c}\text { Ginkgo biloba extract } \\
\text { improved } \\
\text { chemotherapy } \\
\text { sensitivity and } \\
\text { reversed } \\
\text { chemoresistance in } \\
\text { gastric cancer cells by } \\
\text { suppressing the KSR1- } \\
\text { mediated ERK1/2 } \\
\text { pathway }\end{array}$ & {$[92]$} \\
\hline
\end{tabular}


TABle 3: Continued.

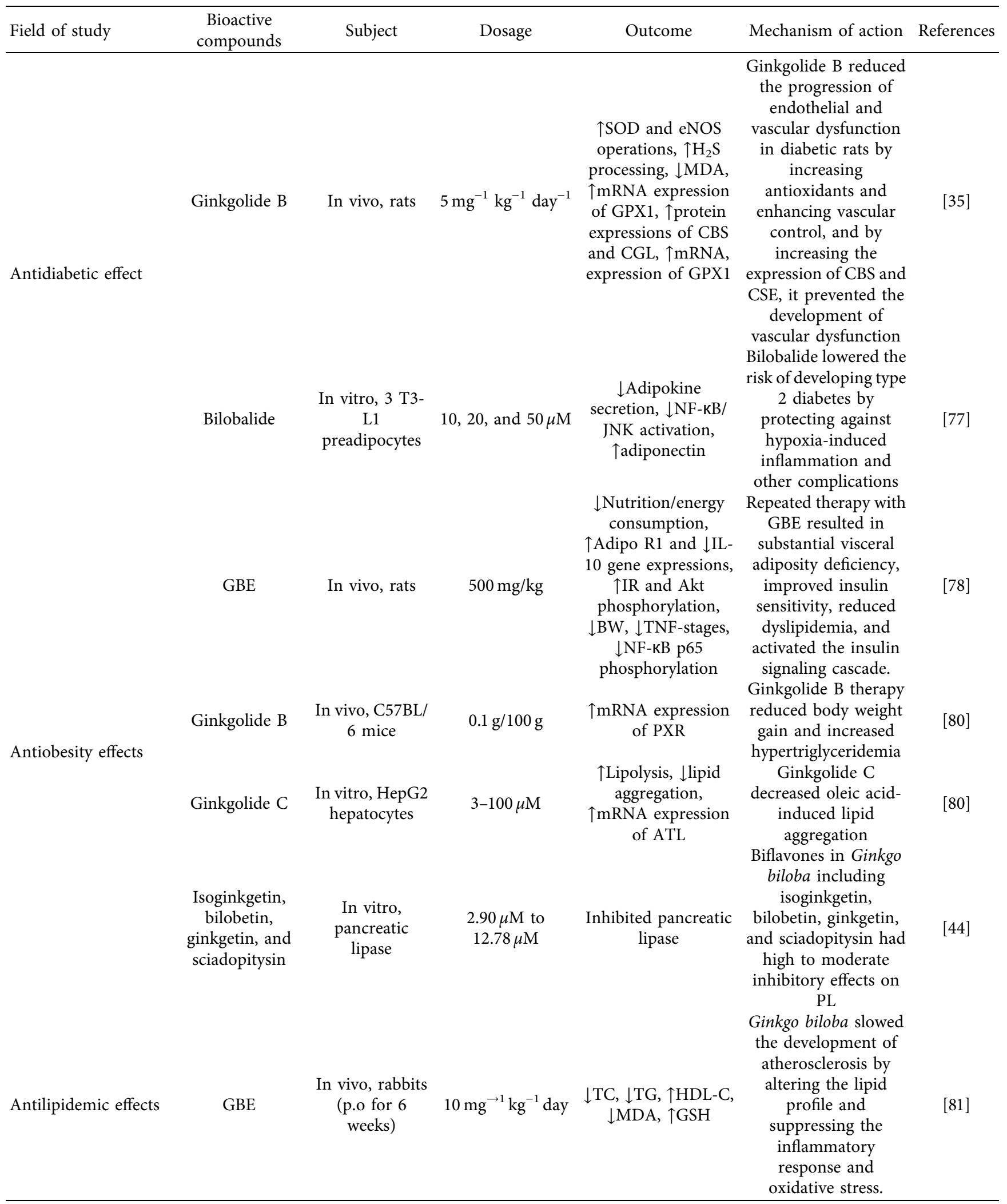


TABle 3: Continued.

\begin{tabular}{|c|c|c|c|c|c|c|}
\hline Field of study & $\begin{array}{l}\text { Bioactive } \\
\text { compounds }\end{array}$ & Subject & Dosage & Outcome & Mechanism of action & References \\
\hline \multirow[b]{2}{*}{$\begin{array}{l}\text { Treatment of } \\
\text { cardiovascular } \\
\text { diseases }\end{array}$} & $\begin{array}{c}\text { Ginkgo seed } \\
\text { ethanol extract }\end{array}$ & $\begin{array}{l}\text { In vivo high-fat } \\
\text { diet (HFD) } \\
\text { mice (p.o. for } 4 \\
\text { weeks) }\end{array}$ & $300 \mathrm{mg} / \mathrm{kg} /$ day & $\begin{array}{l}\downarrow \text { High-fat diet mice’s } \\
\text { epididymal adipose } \\
\text { tissue weight and } \\
\text { adipocyte size, } \\
\downarrow \text { high-density } \\
\text { lipoprotein }\end{array}$ & $\begin{array}{c}\text { Seeds of Ginkgo biloba } \\
\text { provided a } \\
\text { hypocholesterolemic } \\
\text { effect by inhibiting } \\
\text { lipid synthesis, which } \\
\text { may be effective for } \\
\text { the treatment of } \\
\text { cardiovascular } \\
\text { diseases }\end{array}$ & {$[82]$} \\
\hline & $\begin{array}{c}\text { Detoxified } \\
\text { Ginkgo nut } \\
\text { powder (DGP) }\end{array}$ & $\begin{array}{l}\text { In vivo obese } \\
\text { C57BL/6J mice }\end{array}$ & $\begin{array}{c}0.67 \mathrm{mg} / \mathrm{kg} \\
\text { ginkgolic acid, } \\
\text { and } 701.3 \mathrm{mg} / \mathrm{kg} \\
\text { gingko flavonoids }\end{array}$ & $\begin{array}{c}\downarrow \text { Liver and body fat } \\
\text { weights, } \downarrow \text { serum and } \\
\text { hepatic amounts of } \\
\text { triglyceride, } \downarrow \text { low- } \\
\text { density lipoprotein } \\
\text { cholesterol, } \downarrow \text { total } \\
\text { cholesterol, } \downarrow \text { leptin, } \\
\uparrow \text { PPAR } \alpha \text {, } \\
\uparrow \text { hormone-sensitive } \\
\text { lipase, } \uparrow \text { adipose } \\
\text { triglyceride lipase, } \\
\uparrow \text { monoacylglycerol } \\
\text { lipase }\end{array}$ & $\begin{array}{l}\text { Detoxified Ginkgo } \\
\text { biloba nut powder } \\
\text { minimized damage } \\
\text { and increased the } \\
\text { regular lipid } \\
\text { metabolism }\end{array}$ & {$[83]$} \\
\hline Antimicrobial effects & Ginkgolic acids & $\begin{array}{l}\text { Two bacteria } \\
\text { and four fungi }\end{array}$ & $0.11 \mathrm{~g} / \mathrm{kg}$ & $\begin{array}{l}\text { K. pneumoniae had } \\
\text { the maximum } \\
\text { antimicrobial } \\
\text { activity, with a MIC } \\
\text { of } 1.0 \times 10^{5} \mathrm{~g} / \mathrm{mL} \text {, } \\
\text { while } E \text {. coli had the } \\
\text { lowest antimicrobial } \\
\text { activity, with a MIC } \\
\text { of } 7.5 \times 10^{3} \mathrm{~g} / \mathrm{mL} \text {. }\end{array}$ & $\begin{array}{l}\text { Ginkgolic acids had a } \\
\text { wide antimicrobial } \\
\text { repertoire against } \\
\text { bacteria such as E. coli } \\
\text { and Bacillus subtilis, as } \\
\text { well as fungi like } \\
\text { Penicillium } \\
\text { purpurogenum, } \\
\text { Penicillium } \\
\text { camemberti, and } \\
\text { Aspergillus niger }\end{array}$ & {$[93]$} \\
\hline
\end{tabular}


Table 3: Continued.

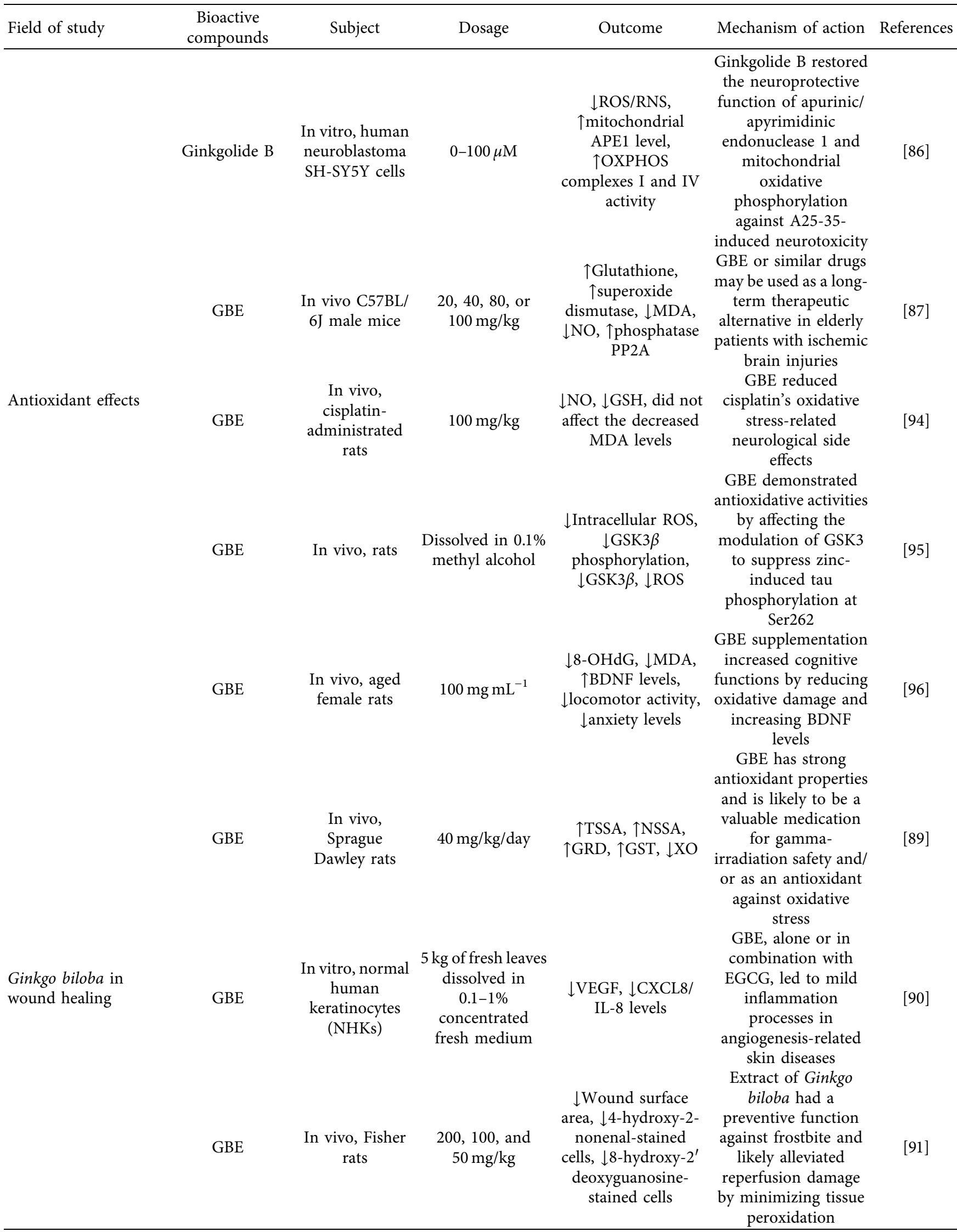


TABle 3: Continued.

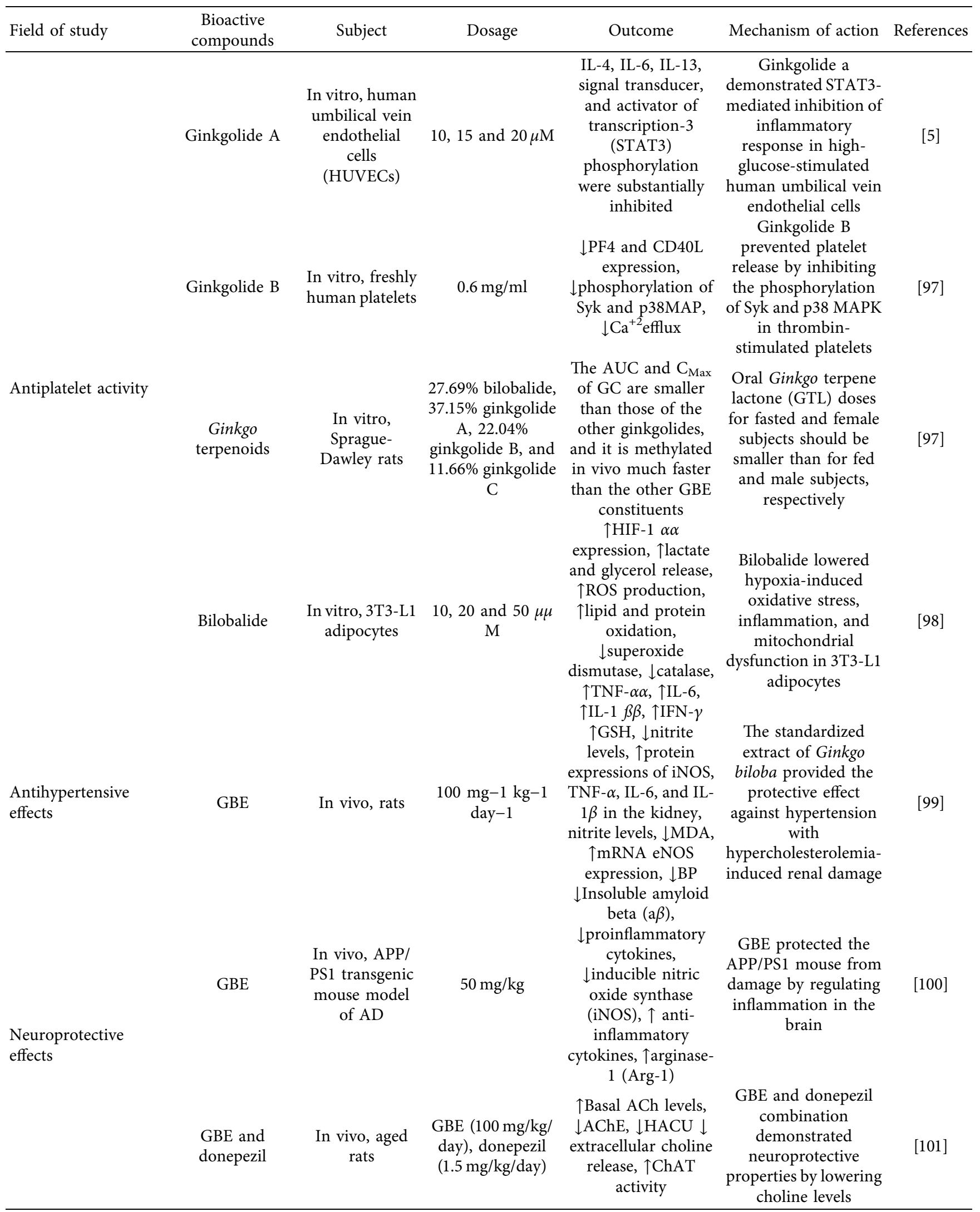


Table 3: Continued.

\begin{tabular}{|c|c|c|c|c|c|c|}
\hline Field of study & $\begin{array}{l}\text { Bioactive } \\
\text { compounds }\end{array}$ & Subject & Dosage & Outcome & Mechanism of action & References \\
\hline Antiaging effect & $\begin{array}{c}\text { Flavonoids, } \\
\text { lactones, } \\
\text { kaempferol 3-O- } \\
\beta \text {-D- } \\
\text { glucopyranoside, } \\
\text { isorhamnetin-3- } \\
\text { O-glucoside, } \\
\text { myricetin, } \\
\text { ginkgolide A, and } \\
\text { bilobalide }\end{array}$ & $\begin{array}{c}\text { In vitro, human } \\
\text { dermal } \\
\text { fibroblasts } \\
\text { (HDFs) }\end{array}$ & n.m. & $\begin{array}{l}\text { Improved skin } \\
\text { condition and } \\
\text { antiaging effect }\end{array}$ & $\begin{array}{c}\text { Ginkgo biloba } \\
\text { extraction impaired } \\
\text { ROS and MMP-1 } \\
\text { degradation in HDFs } \\
\text { because of elevated } \\
\text { levels of flavonoids } \\
\text { and lactones }\end{array}$ & {$[25]$} \\
\hline $\begin{array}{l}\text { Immunomodulatory } \\
\text { effects }\end{array}$ & $\begin{array}{l}\text { Ginkgo biloba in } \\
\text { powder form }\end{array}$ & $\begin{array}{l}\text { In vivo, } \\
\text { rainbow trout } \\
\text { fish }\end{array}$ & $\begin{array}{l}\text { In non-diazinon } \\
\text { fish, } 1 \text { and } 2 \mathrm{~g} \mathrm{~GB} / \\
\mathrm{kg} \text { diet for } 60 \text { days. } \\
\text { In diazinon- } \\
\text { exposed fish, } 0.5 \\
\text { and } 4 \mathrm{~g} \mathrm{~GB} / \mathrm{kg} \text { diet } \\
\text { was fed }\end{array}$ & $\begin{array}{c}\text { Enhanced the } \\
\text { immunity at } \\
\text { optimum dietary } \\
\text { levels }(1-2 \mathrm{~g} / \mathrm{kg} \text { diet }) \\
\text { but showed } \\
\text { immunosuppressive } \\
\text { effects at high dietary } \\
\text { levels }(4 \mathrm{~g} / \mathrm{kg} \text { diet })\end{array}$ & $\begin{array}{c}\text { Ginkgo biloba } \\
\text { increased monoamine } \\
\text { neurotransmitter } \\
\text { levels and altered the } \\
\text { glyphosate-induced } \\
\text { hepatotoxicity, } \\
\text { nephrotoxicity, lipid } \\
\text { peroxidation, and } \\
\text { genotoxicity }\end{array}$ & {$[102]$} \\
\hline \multirow[b]{2}{*}{$\begin{array}{l}\text { Anti-inflammatory } \\
\text { effect }\end{array}$} & $\begin{array}{l}\text { Ginkgo biloba, } \\
\text { ethyl acetate } \\
\text { extract of Ginkgo } \\
\text { biloba, the extract } \\
\text { of total flavonol } \\
\text { glycosides and } \\
\text { full extract of } \\
\text { Ginkgo biloba } \\
\text { seed }\end{array}$ & $\begin{array}{l}\text { In vivo, } 28 \text {-day- } \\
\text { old BALB/c } \\
\text { mice (half } \\
\text { females and } \\
\text { half males) }\end{array}$ & $\begin{array}{l}10 \mathrm{mg} / \mathrm{kg} \text { of body } \\
\text { weight for low } \\
\text { dosage and } 40 \mathrm{mg} / \\
\mathrm{kg} \text { of body weight } \\
\text { for high dosage } \\
\text { form }\end{array}$ & $\begin{array}{c}\text { Showed anti- } \\
\text { inflammatory effect } \\
\text { in airway } \\
\text { inflammation }\end{array}$ & $\begin{array}{l}\text { Ginkgetin reduced the } \\
\text { expression of mRNA } \\
\text { MUC5AC and } \\
\text { regulates pathways } \\
\text { p38 and Akt of the } \\
\text { A549 cell model } \\
\text { stimulating HNE }\end{array}$ & {$[71]$} \\
\hline & $\begin{array}{l}\text { Ethanol extract of } \\
\text { Ginkgo flowers, } \\
\text { bilobetin, } \\
\text { isoginkgetin }\end{array}$ & $\begin{array}{c}\text { In vitro, } \\
\text { RAW264.7 } \\
\text { murine } \\
\text { macrophage } \\
\text { cell }\end{array}$ & $\begin{array}{c}\text { Different } \\
\text { extractive } \\
\text { fractions at } 10 \mu \mathrm{g} / \\
\mathrm{ml} \text { or } 100 \mu \mathrm{g} / \mathrm{ml} \text {, } \\
24 \mathrm{~h} \text { incubation, at } \\
\text { a concentration of } \\
100 \mu \mathrm{M}\end{array}$ & $\begin{array}{l}\text { Effectively inhibited } \\
\text { the generation of } \\
\text { proinflammatory } \\
\text { cytokines and } \\
\text { mediators, making } \\
\text { them valuable for the } \\
\text { treatment of } \\
\text { inflammatory } \\
\text { disease }\end{array}$ & $\begin{array}{c}\text { Bilobetin and } \\
\text { isoginkgetin can } \\
\text { dramatically } \\
\text { downregulate the } \\
\text { levels of NO, TNF- } \alpha \text {, } \\
\text { IL-6, PGE2, iNOS } \\
\text { mRNA, and COX-2 } \\
\text { mRNA, in a dose- } \\
\text { dependent manner, } \\
\text { which may be the } \\
\text { mechanism of their } \\
\text { anti-inflammatory } \\
\text { effects }\end{array}$ & {$[103]$} \\
\hline $\begin{array}{l}\text { Hepatoprotective } \\
\text { effect }\end{array}$ & $\begin{array}{l}\text { Ginkgo biloba } \\
\text { extract }\end{array}$ & $\begin{array}{l}\text { In vivo, male } \\
\text { SD rats }\end{array}$ & $\begin{array}{l}\text { For } 7 \text { days, } 60 \text {, } \\
\text { 120, and } 180 \mathrm{mg} / \\
\text { kg daily dose } \\
\text { before MTX } \\
\text { treatment); } \\
\text { silymarin } \\
\text { (followed by MTX } \\
\text { treatment) }\end{array}$ & $\begin{array}{l}\text { Showed antioxidant } \\
\text { and anti- } \\
\text { inflammatory effects } \\
\text { in methotrexate } \\
\text { (MTX) induce } \\
\text { marked hepatic } \\
\text { injury }\end{array}$ & $\begin{array}{c}\text { Ginkgo biloba extract } \\
\text { reduced expression } \\
\text { levels of TNF- } \alpha \text {, p- } \\
\text { JNK, caspase-3, and } \\
\text { COX-2 pathways in } \\
\text { rat liver }\end{array}$ & {$[104]$} \\
\hline $\begin{array}{l}\text { Anti-depressant } \\
\text { effect }\end{array}$ & $\begin{array}{l}\text { Diterpene } \\
\text { ginkgolides } \\
\text { (DGs) }\end{array}$ & In vivo, mice & $\begin{array}{l}\text { DG }(12.18 \mathrm{mg} / \mathrm{kg}) \\
\text { and venlafaxine } \\
(16 \mathrm{mg} / \mathrm{kg}) \text { for } 14 \\
\text { days }\end{array}$ & $\begin{array}{c}\text { Exhibited } \\
\text { antidepressant-like, } \\
\text { but not anxiolytic- } \\
\text { like, effects }\end{array}$ & $\begin{array}{c}\text { DG reduced } \\
\text { depressive symptoms } \\
\text { and produced } \\
\text { neuroprotective } \\
\text { effects on the } \\
\text { prefrontal cortex by } \\
\text { changing differential } \\
\text { metabolites primarily } \\
\text { involved in amino } \\
\text { acid, energy, and lipid } \\
\text { metabolism }\end{array}$ & {$[105]$} \\
\hline
\end{tabular}


TABLE 4: Clinical studies on therapeutic effects of Ginkgo biloba.

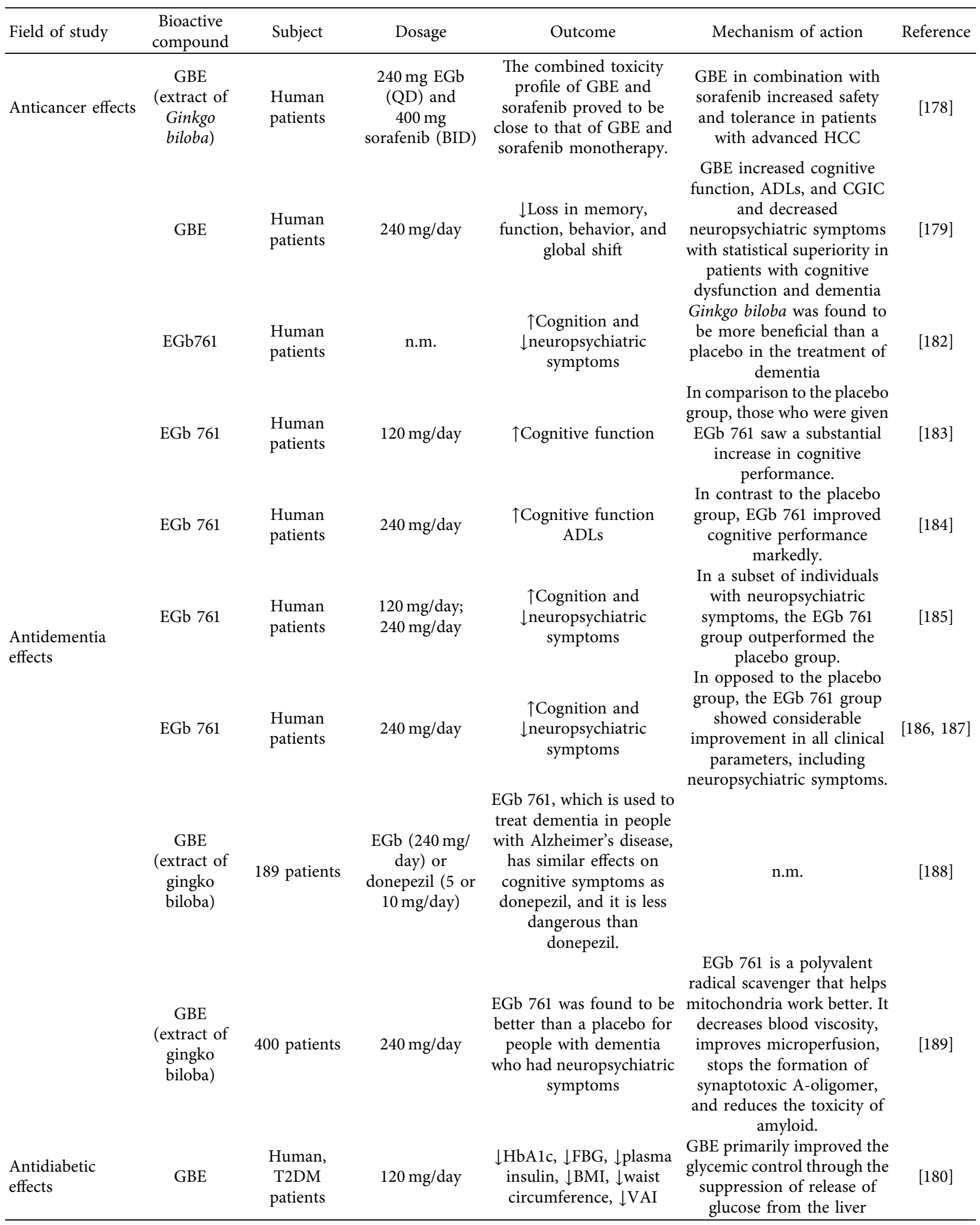


TABle 4: Continued.

\begin{tabular}{|c|c|c|c|c|c|c|}
\hline Field of study & $\begin{array}{l}\text { Bioactive } \\
\text { compound }\end{array}$ & Subject & Dosage & Outcome & Mechanism of action & Reference \\
\hline $\begin{array}{l}\text { Treatment of } \\
\text { tardive dyskinesia }\end{array}$ & $\begin{array}{l}\text { GBE } \\
\text { (extract of } \\
\text { gingko } \\
\text { biloba) }\end{array}$ & $\begin{array}{l}3 \text { human } \\
\text { randomized } \\
\text { controlled trial } \\
(\mathrm{RCT})\end{array}$ & $\begin{array}{l}240 \mathrm{mg} / \text { day, } 12 \\
\text { weeks duration }\end{array}$ & $\begin{array}{c}\text { Reduced the severity of } \\
\text { TD and clinical } \\
\text { symptoms. }\end{array}$ & $\begin{array}{l}\text { GBE scavenges free radicals } \\
\text { and inhibited the formation } \\
\text { of free radicals that reduce } \\
\text { oxidative stress, enhance } \\
\text { BDNF levels, and decrease } \\
\text { the chance of neurotoxicity }\end{array}$ & {$[133]$} \\
\hline $\begin{array}{l}\text { Treatment of } \\
\text { generalized } \\
\text { anxiety disorder } \\
(\mathrm{GAD})\end{array}$ & GBE & $\begin{array}{l}\text { Patients with } \\
\text { GAD (25-30 } \\
\text { patients per } \\
\text { group) }\end{array}$ & $\begin{array}{l}240 \mathrm{mg} \text { and } \\
480 \mathrm{mg} \text { placebo } \\
\text { for four weeks }\end{array}$ & $\begin{array}{c}\text { Improve cognitive } \\
\text { functions and reduce } \\
\text { anxiety under } \\
\text { pathological conditions }\end{array}$ & $\begin{array}{c}\text { Flavonoids in GBE } \\
\text { demonstrated powerful } \\
\text { antioxidant qualities which } \\
\text { helped to reduce anxiety-like } \\
\text { disorders }\end{array}$ & {$[136]$} \\
\hline $\begin{array}{l}\text { Antilipidemic } \\
\text { effects }\end{array}$ & $\begin{array}{l}\text { Ginkgo } \\
\text { biloba } \\
\text { leaves }\end{array}$ & $\begin{array}{l}\text { Human } \\
\text { patients }\end{array}$ & $\begin{array}{l}\text { A variety of } \\
\text { doses }\end{array}$ & $\begin{array}{c}\downarrow \mathrm{TC}, \uparrow \mathrm{HDL}-\mathrm{C}, \downarrow \text { LDL-C, } \\
\downarrow \text { TG }\end{array}$ & $\begin{array}{c}\text { Ginkgo biloba leaves were } \\
\text { more effective than statin } \\
\text { treatment alone in } \\
\text { improving blood lipid } \\
\text { parameters }\end{array}$ & {$[181]$} \\
\hline $\begin{array}{l}\text { Antidepressant } \\
\text { effect }\end{array}$ & $\begin{array}{l}\text { Ginkgo } \\
\text { biloba } \\
\text { extract } \\
\text { (GBE) }\end{array}$ & $\begin{array}{l}136 \text { depressed } \\
\text { elderly } \\
\text { patients }\end{array}$ & $\begin{array}{c}19.2 \mathrm{mg} \text { per time } \\
\text { and } 3 \text { times a } \\
\text { day }\end{array}$ & $\begin{array}{l}\text { Improved depressive } \\
\text { symptoms and decreased } \\
\text { serum S100B expression. }\end{array}$ & $\begin{array}{l}\text { GBE restored neurologic } \\
\text { function and shows the } \\
\text { antidepressant-like effect via } \\
\text { modulation of serotonergic } \\
\text { and dopaminergic } \\
\text { neurotransmission }\end{array}$ & {$[116]$} \\
\hline $\begin{array}{l}\text { Treatment of } \\
\text { vitiligo vulgaris }\end{array}$ & $\begin{array}{l}\text { Ginkgo } \\
\text { biloba BID }\end{array}$ & $\begin{array}{l}12 \text { participants } \\
\text { between } 12 \\
\text { and } 35 \text { years } \\
\text { old }\end{array}$ & $\begin{array}{c}60 \mathrm{mg} \text { of } \\
\text { standardized } \\
\text { G. biloba two } \\
\text { times per day for } \\
12 \text { weeks }\end{array}$ & $\begin{array}{l}\text { G. biloba has significant } \\
\text { improvement in total } \\
\text { vitiligo area scoring index } \\
\text { and vitiligo measures and } \\
\text { vitiligo European Task } \\
\text { force spread }\end{array}$ & $\begin{array}{l}\text { Having anti-inflammatory, } \\
\text { immunomodulatory, and } \\
\text { antioxidant properties, } \\
\text { Ginkgo and its constituents } \\
\text { impact the oxidative stress } \\
\text { mechanisms of vitiligo } \\
\text { decreasing basal } \\
\text { corticosterone secretion, } \\
\text { corticotrophin releasing } \\
\text { hormone (CRH), and } \\
\text { arginine vasopressin (AVP) } \\
\text { gene expression }\end{array}$ & {$[189]$} \\
\hline $\begin{array}{l}\text { Treatment of } \\
\text { ADHD }\end{array}$ & $\begin{array}{l}\text { Ginkgo } \\
\text { biloba } \\
\text { extract } \\
\text { (GBE) }\end{array}$ & 50 patients & $\begin{array}{l}\text { Ginko T.D. }{ }^{\mathrm{TM}} \text { at } \\
\text { a dose of } \\
80-120 \mathrm{mg} / \text { day } \\
\text { for } 6 \text { weeks }\end{array}$ & $\begin{array}{c}\text { G. biloba would be } \\
\text { beneficial for treatment of } \\
\text { ADHD }\end{array}$ & n.m. & {$[190]$} \\
\hline
\end{tabular}

ethanolic extract effectively prevented fat accumulation and lowered the overall body weight in mice, associated with a substantial decrease in adipocyte size and epididymal adipose tissue weight compared with untreated animals [40], suggesting that Ginkgo seed ethanol extract might reduce serum levels of HLD cholesterol. These data indicate that $G$. biloba seeds have a hypocholesterolemic impact via the reduction of lipid synthesis, which might be beneficial for the treatment of cardiovascular disorders [82]. Liu et al. examined the effects of $G$. biloba nut powder on the lipid metabolism in obese mice. The liver weights and serum and hepatic levels of LDL cholesterol, leptin, TG, and total cholesterol in obesity model mice fed G. biloba nut powder were equivalent to those of control mice but substantially lower than those in the untreated model group. They also indicated that detoxified $G$. biloba nut powder might decrease damage and enhance the lipid metabolism [83]. The therapeutic potentials of $G$. biloba are shown in Figures 3(a)3(b)).
5.8. Antimicrobial Effects. Antimicrobial activity has been established for several components of G. biloba plants, including phenolic acids, polysaccharides, and proteins. Ginkgolic acids have been the subject of several experiments examining the antimicrobial functions of $G$. biloba seeds. Ginkgolic acids are fatty acids with the compositions C13:0, $\mathrm{C} 15: 0, \mathrm{C} 15: 1, \mathrm{C} 17: 1$, and $\mathrm{C} 17: 2$, and the antimicrobial activities of ginkgolic acids are aided by the presence of an alkyl side-chain [40]. Ginkgolides have been shown to be bactericidal against Staphylococcus aureus, Bacillus cereus, and Bacillus subtilis, but not against Escherichia coli. Ginkgolic acids, by contrast, have demonstrated potent antibacterial activity against $E$. coli, with a minimum inhibitory concentration (MIC) of $7.5 \times 10^{-3} \mathrm{~g} / \mathrm{mL}$. Ginkgolides also demonstrated antimicrobial activity against Penicillium and B. subtilis, with MIC values of $2.5 \times 10^{-4} \mathrm{~g} /$ $\mathrm{mL}$ and $1.5 \times 10^{-4} \mathrm{~g} / \mathrm{mL}$, respectively. Wu et al. identified a protein with broad-spectrum antibacterial activity in G. biloba plants using an ammonium sulfate precipitation. 
Some Gram-negative and Gram-positive bacteria were inhibited by the identified protein, with the strongest activity against Klebsiella pneumoniae, with an MIC of $1.0 \times 10^{-5} \mathrm{~g}$ / mL. An MIC of $1.0 \times 10^{-5}$ was also identified against Torulaspora delbrueckii, Penicillium, and Aspergillus niger. Polysaccharides comprised $50 \%-80 \%$ of the ethanol precipitation of $G$. biloba seeds, and $87.4 \%$ of polysaccharides and $1.0 \%$ of proteins displayed antibacterial characteristics [84].

5.9. Antioxidant Effects. Tissues undergo DNA oxidative stress, oxidative protein injury, lipid peroxidation, and other oxidative injuries when they mature. Neurological activity, sensory tissues, and the cardiovascular system may all be affected as a result [84], and these defects can have significant influences on the progression of degenerative diseases [85]. $G$. biloba has several distinct antioxidant components, making it difficult to test each antioxidant independently. In diseased tissues, higher quantities of free radicals and oxidatively damaged lipids, DNA, and proteins and reduced levels of antioxidants have all been reported. Terpenes, flavonoids, and bioflavonoid components are thought to contribute the antioxidant capabilities of G. biloba, which has been shown to be beneficial against a variety of free radical-generating substances, including oxyferryl, superoxide, hydroxyl, NO, and peroxyl radicals. The antioxidant properties of $G$. biloba contribute to protecting the cardiovascular system, brain, and retina from free radical damage associated with aging. The antioxidant capabilities and flavonol contents of $G$. biloba leaf crude extracts were investigated [86], and Kaur et al. found that ginkgolide B derived from EGb 761 applied to human neuroblastoma IMR-32 and SHSY5Y cells reduced ROS/RNS production by prooxidant A25-35 peptides [86]. MDA and NO levels increased with age but were suppressed by pretreatment with EGb 761. By controlling oxidative stress, EGb 761 can protect against ischemic injury [87]. Aydin utilized cisplatin to promote oxidation in the rat brain and observed that EGb 761 reduced GSH and NO levels in brain tissue, which reduced oxidative stress [7].

5.10. Wound Healing. Wound healing is a biochemical mechanism that repairs weakened tissues and preserves skin integrity. Hemostasis, inflammation, proliferation, and remodeling are the four phases of skin wound healing. Cytokines promote healing through various methods, including boosting basement membrane material production, avoiding dehydration, enhancing inflammation, and hastening the formation of granulated tissues [88]. Okumus et al. examined the antioxidant impacts of $G$. biloba on the prevention of radiation-induced cataract development in the rat lens. In the presence of radiation, GBE significantly enhanced total (enzymatic and nonenzymatic) superoxide scavenger activity (TSSA), glutathione-S-transferase (GST), glutathione reductase (GRD), and nonenzymatic superoxide scavenger activity (NSSA). In the irradiation + GBE groups, the activity of lens xanthine oxidase (XO) was reduced substantially [89]. In cranium-irradiated studies, G. biloba increased the activities of SOD and GPX while decreasing lipid peroxidation activity [90]. Frostbite occurs when tissues are exposed to cold temperatures, commonly resulting in freezing on the extremities and can result in the need for amputation or cause tissue failure and impairment, associated with the development of free radicals, which cause additional tissue damage. Dong reported that EGb 761 treatment was able to reduce frostbite damage as a free radical scavenger [91]. The in vivo and in vitro studies examining the therapeutic effects of $G$. biloba are given in Table 3.

5.11. Antiplatelet Activity. Platelet-activating factor (PAF) is a phospholipid-derived messenger that contributes to the immunological response to infection and neuronal damage induced by ischemia and excitotoxic stress. PAF is produced in response to inflammatory damage and can serve as a paracrine, endocrine, or autocrine messenger by activating the PAF receptor (PAFR) to activate inflammatory proteins [75]. The PAFR is associated with the Janus kinase (JAK)/ signal transducer and activator of the transcription (STAT) signaling pathway, which regulates gene transcription in response to cytokines and growth factors [5]. The release of CD40L and regulated upon activation, normal $\mathrm{T}$ cell expressed and presumably secreted (RANTES) by platelets activated by thrombin and collagen is significantly reduced by treatment with $0.6 \mathrm{mg} / \mathrm{mL}$ ginkgolide $\mathrm{B}$. In thrombin $(0.5 \mathrm{U} / \mathrm{mL})$-activated platelets, ginkgolide $\mathrm{B}(0.6 \mathrm{mg} / \mathrm{mL})$ suppressed ATP release by $50.8 \%$ and partially reduced calcium efflux by $52.7 \%$, according to a recent study. Ginkgolide B was reported to reduce platelet release by decreasing the phosphorylation of p38 mitogen-activated protein kinase (MAPK) and Syk [97]. As a PAF antagonist, ginkgolide $\mathrm{C}$ was found to be 25 times less effective than ginkgolide $\mathrm{B}$ due to the presence of the $7-\mathrm{OH}$ replacement, which is absent from other ginkgolides. Ginkgolide $C$ had lower area under the curve (AUC) and peak serum concentration $\left(C_{\max }\right)$ than the other ginkgolides and is methylated in vivo much faster than the other EGb 761 components, which might explain the overall reduced activity [97]. In addition to its impact on PAF, bilobalide, like ginkgolides, offers additional health benefits. Bilobalide's anti-inflammatory effects are supported by a new study that shows it reduces hypoxia-induced inflammation and discomfort [98].

5.12. Anti-Inflammatory Effects. Inflammation is a complicated biochemical process that leads to the destruction of tissue homeostasis. Inflammation can be induced by various molecular, mechanical, or physical agents and can be classified as chronic or acute [106]. Inflammation is typically triggered by the release of substances from tissues and migratory cells, such as histamine, prostaglandins (PGs), leukotrienes (LTs), bradykinin, PAF, and IL [107]. Various cytokines and chemokines produced by dendritic cells and macrophages stimulate circulating leukocytes after they enter the local injury site. Neutrophils phagocytose antigens, releasing ROS and cytokines, include IL-1, IL-6, and TNF- $\alpha$ 
[108]. Polyphenolic chemicals found in G. biloba, such as flavonoids, have been shown to have anti-inflammatory properties [109]. Coughs, asthma, and other lung disorders have long been treated with G. biloba in traditional Chinese medicine. In an ovalbumin-induced allergy mouse model, the pharmacological effects of several GBEs were examined. The ethyl acetate phase of the extract included anti-inflammatory compounds, which were identified using highperformance liquid chromatography (HPLC)-MS. The primary compounds were identified as biflavones, one of which ginkgetin has a good fit score with leukocyte elastase, and the effects of biflavones were studied in both in vivo and in vitro settings. In human neutrophil elastase (HNE)-stimulated A549 cells, ginkgetin significantly decreased the abnormal expression of the Akt and p38 pathways. Mucin 5AC (MUC5AC) mRNA expression was also reduced by biflavones in HNE-stimulated A549 cells and the allergic mouse modes, and biflavone-treated mice showed reduced levels of inflammatory cells and cytokines. These data suggested that G. biloba biflavones may decrease leukocyte elastase activity, and G. biloba has been identified as a functional food for the treatment of airway inflammation [71]. In another study, LPS-induced RAW264.7 macrophages treated with $100 \mathrm{~g} /$ $\mathrm{mL}$ of the ethanol extract of $G$. biloba flowers or the chloroform and ethyl acetate fractions showed greatly reduced prostaglandin E2 (PGE2), NO, and IL-6 outputs. Bilobetin and isoginkgetin, the most powerful compounds identified in GBEs, increased the NO inhibition ratios to $80.19 \%$ and $82.37 \%$ at $50 \mathrm{M}$, respectively. They also inhibited TNF- $\alpha$, IL-6, PGE2, and cyclooxygenase 2 mRNA levels in a dose-dependent manner, indicating that they may be promising candidates for the discovery of new anti-inflammatory drugs [103].

5.13. Hepatoprotective Effects. The antioxidant activity of G. biloba is thought to be responsible for its hepatoprotective benefits, associated with the restoration of SOD, GPX, and catalase (CAT) activities, in addition to increasing glutathione contents and reducing the levels of lipid peroxidation and hydroperoxides in the liver [110]. The hepatoprotective effect of GBEs in rats with obstructive yellowing was also examined $[69,111]$, revealing that GBE greatly improved serum transaminase levels and reduced liver histological damage, resulting in increased liver defense. Pretreatment with GBE prevented the release of TNF- $\alpha$ and upregulation of IL- 6 mRNA. In addition, the necrotic region of the central lobe was diminished by GBE therapy $[69,112]$. G. biloba has hepatoprotective effects against carbon tetrachloride $\left(\mathrm{CCl}_{4}\right)$ induced hepatic oxidative injury in rats [113]. G. biloba phytosome (GBP) may act through an initial decrease in hepatic peroxidative activities, accompanied by the inhibition of glutathione (GSH)-related enzymes [67]. The antioxidant and hepatoprotective properties of GBE have been shown to prevent liver fibrosis in rats [114].

5.14. Antidepressant Effects. Depression is a widespread and long-lasting mental disorder that affects mood and feelings, resulting in global economic losses. Gut microbiota, especially probiotics, have been shown to influence the development of depression and recovery. Polysaccharides have antidepressive effects through the regulation of the microbiota-gut-brain axis $[110,115]$. GBE can effectively reduce depressive symptoms and suppress serum S100B expression, which suggests that GBE restores the neurological activity in older adults treated for. A synergistic effect is observed when GBE is combined with antidepressant drugs, resulting in more rapid results than the use of single antidepressant drugs alone [116]. A water-soluble G. biloba polysaccharide reduces depression caused by stress and reverses intestinal dysbiosis. GPS mice had higher serotonin and dopamine levels than the unpredictable chronic moderate stress mice in many brain areas. The polysaccharides derived from G. biloba leaves may represent successful pharmacological candidates for depression treatment [117]. GBE resulted in a strong increase in venlafaxine in PSD treatment [118]. Diterpene ginkgolides (DGs) in GBE have been shown to exert neuroprotective effects across many previous studies. DGs have antidepressant but not antianxiety effects in mice, indicating that GBE might be useful for the treatment of major depressive disorders [105]. Chronic DG therapy greatly improves the characteristics of depression [119]. In mice with heart disease, GBE increased antidepressant-like activities. TNF- $\alpha$, IL-1, and 5-HT levels in the hippocampus were decreased after GBE administration. GBE also inhibited serotonin release in peripheral blood and activated hypoxia-inducible factor 1 (HIF-1)associated antiapoptotic pathways [120]. Compared with control groups, pretreatment with 30 and $60 \mathrm{mg} / \mathrm{kg} /$ day GBE substantially reduced depressive-like effects in RSS rats and prevented changes in DNA methylation and protein expression of the brain-derived neurotrophic factor (BDNF) in the hippocampus induced by persistent stress. These results indicate that GBE may exert antidepressant effects by modulating BDNF expression in the hippocampal nucleus [121].

5.15. Antiaging Effects. Skin ages primarily due to the effects of oxidative stress. Aging skin, increased collagen loss, and poor desquamation can be distinguished by increased wrinkles and a dry and dull appearance. G. biloba leaf extract and aqueous $G$. biloba ethanol extract have been shown to display skin protective effects, and the molecular pathways involved have been studied using $\mathrm{HaCaT}$ keratinocytes, revealing antioxidant effects and antiaging results [122]. Many different GBEs have been shown to cause biphasic reactions in a wide variety of cell types. GBE applied as a topical formula achieved good skin penetration and retention, with beneficial effects for the skin, including protection from ultraviolet (UV) damage [123]. EGb 761 treatment in rats showed a preventive function against frostbite and likely alleviated reperfusion damage by minimizing tissue peroxidation [91], suggesting that EGb 761 may serve as an antiaging agent. However, the antiaging effects of EGb 761 remain inconclusive, particularly the effects on the CNS [124]. Total lactones of Ginkgo (TLG) applied to aged mice displayed antiaging effects by attenuating lipid peroxidation, 
NO levels, and cerebral apoptosis [125]. The main components of EGb 716 can regulate numerous pathways and exert antiaging effects by inhibiting inflammation, reducing oxidative stress, and improving insulin resistance, mediated by several targets, such as PPARG, DPP4, and GSK3B [126].

5.16. Immunomodulatory Effects. Immunomodulators regulate the immune system. Most herbs defend the body against infection through two basic mechanisms: destroying pathogens or increasing immunity [127]. G. biloba polysaccharides (GBPS) exert enhanced antitumor and immune responses. GBPS-2 and GBPS-3 greatly improved macrophage phagocytosis and encouraged NO, TNF- $\alpha$, IL- 1 , and IL- 6 activity, suggesting that GBPS- 2 and GBPS- 3 may serve as useful food supplements for boosting immunity [128]. One study examined the immunostimulatory effects of dietary G. biloba (GB) on oxidative stress and toxicity induced by the organophosphate insecticide diazinon. Plasma total immunoglobulin, lysozyme activity, and peroxidase activity were substantially higher in non-diazinon-exposed fish 60 days of feeding with 1 and $2 \mathrm{~g} \mathrm{~GB} / \mathrm{kg}$. In a group fed $0.5 \mathrm{~g}$ $\mathrm{GB} / \mathrm{kg}$, pulmonary burst activity and complement activity were considerably enhanced. Throughout the feeding study, peroxidase activity, immunoglobulin activity, and lysozyme activity were considerably decreased in the group fed with $4 \mathrm{~g} \mathrm{~GB} / \mathrm{kg}$. No substantial changes in renal IL- $1 \beta$ or transforming growth factor beta-1 (TGF $\beta$-1) gene expression were observed between the non-GB-supplemented fish and GB-supplemented fish. Both immunological components were reduced significantly in diazinon-exposed fish fed with and without 0.5 and $4 \mathrm{~g} \mathrm{~GB} / \mathrm{kg}$ diets. No differences in immunity components were observed for fish fed 1 and $2 \mathrm{~g}$ $\mathrm{GB} / \mathrm{kg}$ diets during the course of the experiment. Diazinon exposure increased the expression of IL- $1 \beta$ and TGF $\beta-1$ genes in fish fed 0.5 and $4 \mathrm{~g} \mathrm{~GB} / \mathrm{kg}$ diets. These findings indicated that GB displayed an immunogenic effect against diazinon toxicity at optimal dietary levels (1-2 g GB/ $/ \mathrm{kg}$ ), but high dietary levels $(4 \mathrm{gGB} / \mathrm{kg})$ resulted in immunosuppressive effects, suggesting that dietary levels must be optimized to ensure beneficial effects [102]. G. biloba exocarp polysaccharides (GBEP) were found to exert effects on cellular immunity and humoral immunity in a mouse immunosuppressive model [129]. Therefore, GBE can be a rapid, healthful, and somewhat effective therapy for halting disease progression and increasing immunity when intake levels are optimized [130].

5.17. Treatment of Tardive Dyskinesia. Tardive dyskinesia (TD) is a common adverse reaction to antipsychotic drugs, which are commonly used to treat dementia and other serious psychiatric illnesses. TD is characterized by uncontrollable, stiff, jerky facial and body motions, resulting in involuntary movements, such as blinking one's eyes, sticking out one's tongue, or waving one's arms [131]. Although low BDNF has been indicated in the pathophysiology of TD, the etiology remains inconclusive, and no well-accepted treatments have been developed. The administration of EGb 761 results in increased BDNF levels through antioxidant mechanisms, which have neuroprotective effects, suggesting that EGb 761 could potentially reduce TD symptoms [132]. A randomized controlled trial (RCT) was performed to assess the antioxidant and free radical-scavenging properties of EGb 761, in which the Abnormal Involuntary Movement Scale was used to quantify the severity of TD symptoms as the primary outcome. EGb 761 (240 mg/day) outperformed the placebo for minimizing the incidence of TD symptoms. A meta-analysis revealed that adjunctive EGb 716 appears to be an efficient and secure alternative for improving TD in schizophrenia patients. However, further RCTs remain necessary to demonstrate the efficacy and protective abilities of adjunctive EGb 716, particularly in terms of protecting the cognitive capacity, in cases of TD [133]. EGb 761 appears to be an appropriate therapy for reducing TD effects in schizophrenic patients, which is likely mediated by the wellknown antioxidant activity of the extract [134].

\subsection{Treatment of Generalized Anxiety Disorder.} Generalized anxiety disorder (GAD) is marked by constant and excessive worry over a variety of topics. Individuals who suffer from GAD expect the occurrence of constant catastrophic events that manifest as excessive concern regarding income, health, family, jobs, and other matters. Individuals who suffer from GAD often struggle to contain their worries [135]. Globally, anxiety and mental illness are the most commonly diagnosed conditions, and various medications have been used to treat mental conditions, including dementia, depression, and anxiety disorders, including GBE and other G. biloba products, which are used globally [136]. The literature suggests that GBE can offer beneficial effects on ischemia, hypoxia, and stress reduction in cases of cognitive deterioration $[137,138]$. EGb 761 improves emotional function and stabilizes mood in older adult patients with cognitive impairment. In addition, EGb 761 has been shown to relieve anxiety symptoms among people with mental deterioration, including in younger patients with anxiety symptoms [139]. The chemical structures and synergy of various chemical components found in EGb 761 are capable of producing neuroprotective effects, mediated by antioxidant effects and the regulation of neurotransmission, neuroendocrine signaling, and neurotrophic factors [140], resulting in the alleviation of anxiety symptoms [141].

5.19. Antihypertensive Effects. Hypertension is a progressive condition that can lead to serious problems, such as acute coronary syndromes, chronic cardiac failure, and stroke [142]. Inflammation, the dysregulation of the renin-angiotensin pathway, autoimmune vascular malfunction, and oxidative stress are among the pathophysiological etiologies associated with the development of hypertension [143]. The antihypertensive effects of $G$. biloba have been observed in a variety of animal models. In a study of rats with impaired kidneys, EGb 761 exerted hypotensive and renoprotective effects by inhibiting renal NO overproduction and reducing IL-6 and TNF- $\alpha$ levels in kidney tissue [99]. NO lowers blood pressure by dilating blood vessels, and the hypotensive effect of GBE may be due to increased eNOS expression and 
NO production. GBE also increases endothelial intracellular $\mathrm{Ca}^{2+}$ levels and endothelium-dependent vasodilation, leading to hypotension [59].

5.20. Neuroprotective Effects. The rapid progression of neurodegenerative diseases occurring within the general population has highlighted the increased need for research to identify the pathogenesis of neurodegenerative disease and develop alternative therapies. Excitatory toxicity is a primary cause of intracerebral neuron death. Neurodegenerative disorders may be prevented by enhancing the cerebral blood supply. G. biloba prevents pathological hyperactivity, allowing the body to reclaim its natural physiological equilibrium. In addition, terpenoid and flavonoids in G. biloba seeds have been shown to improve blood circulation in the brain [40]. GBE has been shown to improve a variety of ailments including $\mathrm{AD}$ and brain ischemia, two age-related neurodegenerative diseases that are important causes of death and morbidity among older adults [144]. The increased occurrence of cerebrovascular disease has been associated with chronic inflammatory disorders [145]. AD is a neurological disease affecting people as they age and results in cognitive impairment [146]. Both immune components in diazinon-exposed fish in control and those fed 0.5 and $4 \mathrm{~g}$ $\mathrm{GB} / \mathrm{kg}$ diets reduced significantly throughout the exposure. Immunity components in fish fed 1 and $2 \mathrm{~g} \mathrm{~GB} / \mathrm{kg}$ diets did not change over the exposure period. Furthermore, diazinon increased the expression of the IL-1 and TGF-1 genes in fish fed 0.5 and $4 \mathrm{~g} \mathrm{~GB} / \mathrm{kg}$ diets. Finally, at optimal dietary levels (1-2 g GB/kg diet), GB has an immunogenic effect against diazinon toxicity. However, GB demonstrated immunosuppressive effects at high dietary levels (4 g GB/kg intake), indicating that its levels in the diet should be optimized [100].

\section{Commercial Use}

G. biloba leaves are used in traditional Chinese medicine to treat neurological problems, circulatory disorders, and respiratory diseases. G. biloba leaves are also used as insecticides and fertilizers [147]. G. biloba leaf extracts are used as food additives due to potential medicinal benefits, which have captivated the global market due to the potential to improve wellbeing [148]. Pharmaceutically bioactive compounds have been identified in G. biloba leaf extracts, including glycosides and ginkgolides. EGb 761 is a common extract used in several countries, including the United States, Germany, China, and France, and is marketed in Europe as a drug for the treatment of cardiovascular disease. EGb 761 is distributed as a dietary supplement to the diet under the trade name Ginkgold. The use of G. biloba components, such as ginkgolides $\mathrm{A}, \mathrm{B}, \mathrm{C}, \mathrm{J}$, and $\mathrm{M}$, is increasing at a rapid pace [4]. Ginkgo shells, which are produced as industrial byproducts at a high annual rate, have not been thoroughly studied. Lignin is an abundant component of Ginkgo shells, including high yields of ferulates, p-coumarates, and vanillin, and is found at higher levels than in most softwoods and hardwoods, making Ginkgo shells a useful source for food and drink flavoring agents. It also has excellent antioxidant functions, with an optimum radical scavenging index of 6.9, higher than industrial antioxidants butyl hydroxyanisole and butylated hydroxytoluene [149]. Ginkgo nuts have historically been used to treat coughs, sputum, fever, diarrhea, toothaches, skin diseases, gonorrhea, and overactive bladder. Ginkgo nuts have also been eaten as a side dish [147]. G. biloba seeds have a hypocholesterolemic effect on the lipid metabolism [150], and EGb 761 has been shown to inhibit diabetic-induced cataracts under rat lenses cultured in high-glucose conditions [82].

\section{Synergistic Effects}

Synergism is the phenomenon in which treatment with two or more agents simultaneously result in more influential health outcomes than would be expected from the sum of the individual effects [151]. By regulating ROS production and lectin-type oxidized LDL receptor 1 (LOX-1) and p38 MAPK expression in human ex vivo endothelial artery cells, therapeutic doses of GBE and aspirin have been demonstrated to have synergistic effects reducing oxidative stress induced by activated platelets [152]. GBE inhibited platelet aggregation induced by tertbutyl hydroperoxide and $\mathrm{Fe}^{2+}$ in a dose-dependent manner. These findings suggest that GBE can inhibit oxidative stress-stimulated platelet aggregation in the brain and myocardial systems [153]. Polyprenols isolated from lipids are newly identified components found in G. biloba leaves. Petroleum ether extraction, saponification, and molecular distillation were used to extract Ginkgo lipids. Previous investigations have examined the antibacterial and antifungal properties of GBP and the chemicals isolated from GBL lipids against Staphylococcus aureus, Aspergillus niger, or Salmonella enterica and their synergistic interactions. The highest efficacy was identified for nerolidol, and the combination of GBP and isophytol exhibited the best synergistic activity against Salmonella enterica among the three strains examined [154]. RCTs have examined the use of GBE for periods ranging from 2 weeks to 2 years, with dosages varying from 80 to $720 \mathrm{mg} / \mathrm{d}$. Interactions have been identified with inhibitors of monoamine oxidase, alprazolam, haloperidol, warfarin, and nifedipine [155]. The combination of isorhamnetin and caffeic acid facilitates the expression of CAT and SOD enzymes in E. coli, which may be due to the synergistic effects between C-3 and EGb 761 [156]. The formulation of green tea and GBE has shown success for the improvement of skin conditions, and the FBlend solution results in more pronounced improvements in skin elasticity [157].

\section{Adverse Effects}

Although $G$. biloba has the potential to treat a variety of diseases, some drawbacks have also been identified. Restani et al. recently explored the negative impacts of plant food additives and botanical preparations and observed that 39 plants species, representative of $59 \%$ of all plants tested, had negative impacts on people. The fourth most hazardous species was determined to be G. biloba [158]. 
8.1. G. biloba Seeds. Tonic and clonic convulsions, vomiting, and loss of consciousness are symptoms associated with G. biloba seed poisoning. The neurotoxic chemical MPN, also known as ginkgotoxin, and its derivative MPN glucoside are the primary sources of toxicity. MPN is structurally similar to vitamin B6 and hinders the synthesis, metabolism, and activities of vitamin B6 [159]. MPN inhibits the enzymatic activation of vitamin B6 by pyridoxal kinase, resulting in vitamin $\mathrm{B} 6$ deficiency and decreased $\gamma$-aminobutyric acid (GABA) synthesis. MPN values in G. biloba seeds range from 170 to $404 \mathrm{ppm}$. A 23-month-old child in Switzerland suffered two afebrile tonic-clonic seizures after swallowing an unknown quantity of G. biloba seeds, and the toxicity was verified by monitoring MPN levels in both blood and urine [160]. A 51-year-old Korean woman who ingested $1 \mathrm{~kg}$ of G. biloba nuts within 1 hour experienced tonic-clonic seizures 12 hours later, associated with postictal confusion and a serum vitamin B6 level of $2.2 \mathrm{~g} / \mathrm{L}$ [161].

8.2. G. biloba Leaf Extracts. Using modern methodologies, MPN identified in G. biloba leaf extract is below the typical detection limit $(9 \mathrm{ppm})$, although the potential that leaf extracts may be contaminated by G. biloba seeds or fruits cannot be entirely excluded. In individuals receiving medications metabolized by CYP2C19 and CYP2C9, GBE consumption should be monitored. More studies remain necessary to determine how MPN interacts with medications that are CYP3A4 or P-gp substrates [162]. Standardized $G$. biloba leaf extract has been examined for the potential to increase the risk of bleeding [163], and the literature regarding bleeding risks has been contentious, although additional case studies continue to be released $[164,165]$. When combined with warfarin, G. biloba leaf extract significantly reduced the risk of bleeding, according to one study of a huge medical record repository in the US [166]. Other G. biloba leaf extract adverse effects have also been recorded. For example, a 35-year-old woman experienced irregular nocturnal palpitations that were connected to the proarrhythmic activities of G. biloba, and a 66-yearold woman experienced allergic contact dermatitis after contact with Ginkgo tree fruit amid Ginkgo leaves [167, 168].

\section{Toxicity}

High doses and long-term administration of G. biloba can cause several toxic effects, as demonstrated in both in vivo and in vitro studies.

9.1. In Vivo Toxicity. G. biloba toxicity has indeed been studied in vivo in rats, in addition to clinical trials. The evaluation of genotoxic and carcinogenic potential represents significant toxicity endpoints during the safety assessment of a drug [169]. In rats and mice of both sexes, a three-month G. biloba treatment increased liver weights and the rate of hepatocytic hypertrophy, and male rats presented with a considerably increased frequency of hepatocellular adenomas. If the dose is continued for 2 years, the survival rate for both male rats and female mice decreased. Another hepatogenesis mechanistic analysis revealed that substantial variations in molecular changes were found between spontaneous and ginkgo-induced HCC in B6C3F1 mice, although these carcinomas were morphologically indistinguishable [170]. The expression of genes associated with Nrf2-mediated oxidative stress and drug metabolic enzymes in the Myc gene-centered network termed "cell cycle, cancer, and cellular mobility" were found to differ significantly in the livers of male B6C3F1 mice based on microarray analysis [171]. During pregnancy, the treatment of female mice with G. biloba capsules at a dose of $100 \mathrm{mg} / \mathrm{kg} /$ day resulted in the significant incidence of abnormalities in mouse fetuses [172]. Polyprenols are common active lipids with neither acute nor subchronic toxicity in rats, which have been found in $0.35 \%$ of dried Ginkgo leaves. The toxicity of G. biloba was less evident during pregnancy. A standardized EGb 761 extract with specified active component concentrations of ginkgolic acids had no influence on embryo-fetal development, preimplantation, or postimplantation losses in CD-1 mice fed $100-1,225 \mathrm{mg} / \mathrm{kg} /$ day EGb 761 daily from day 6 to day 15 of pregnancy $[162,173]$.

9.2. In Vitro Toxicity. The National Toxicology Program (NTP) assessed the genotoxicity of G. biloba leaf extract containing comparable components of known items using bacterial gene mutation tests. Extract (1-10 mg/plate) was mutagenic in Salmonella typhimurium strains TA98 and TA100 and in the E. coli strain WP2 uvrA/pKM101 [174]. The extract also increased intracellular ROS while decreasing GSH levels [175]. At a concentration of $40 \mathrm{M}$ concentration, kaempferol and quercetin inhibit cellular growth in oral cancer cell lines [176]. Picrotoxin, a well-known $\mathrm{GABA}_{\mathrm{A}}$ receptor antagonist, resembles ginkgolides [177]. The resazurin reduction test revealed that three large ginkgolic acids with different alkyl or alkenyl groups $(13: 0,15: 1$, and $17: 1)$ were cytotoxic at $50 \mathrm{M}$ in male Chinese hamster lung fibroblast-like V79 cells.

\section{Clinical Studies}

Clinical trials refer to experimental processes conducted on humans to determine the efficacy and safety of bioactive compounds, such as those found in G. biloba. Cai et al. observed that the maximal combination of $240 \mathrm{mg}$ EGb (QD) and $400 \mathrm{mg}$ sorafenib (BID) was effective and tolerable for HCC patients in a clinical trial assessing the safety and efficacy combined treatment for patients with advanced HCC [178]. Clinical studies examining $240 \mathrm{mg}$ daily EGb 761 administration to dementia patients have indicated that EGb 761 can stabilize or delay mental functional decline, especially among individuals with neuropsychiatric symptoms [179]. A clinical experiment was conducted to examine the antidiabetic efficacy of GBE when combined with metformin as adjuvant therapy. In a 3-month controlled, multicenter, double-blinded study, 60 T2DM patients were randomly assigned to one of two classes (GBE $120 \mathrm{mg}$ /day or placebo (starch) $120 \mathrm{mg} /$ day). The administration of GBE dramatically decreased fasting plasma glucose levels, insulin levels, 
blood HbA1c values, waist circumference, body mass index, and visceral adiposity index [180]. Increased very-low-density lipoprotein (VLDL) secretion by liver cells leads to insulin tolerance and hyperlipidemia [59]. Adjuvant G. biloba treatment improved total cholesterol (median: $0.61 \mathrm{mmol} / \mathrm{L} ; 95 \%$ confidence interval (CI) $0.33-0.90 \mathrm{mmol} / \mathrm{L}), \mathrm{LDL}-\mathrm{C}$ (median: $0.32 \mathrm{mmol} / \mathrm{L} ; 95 \%$ CI $0.16-0.48 \mathrm{mmol} / \mathrm{L}$ ), HDL-C (median: $0.26 \mathrm{mmol} / \mathrm{L} ; 95 \%$ CI $0.15-0.37 \mathrm{mmol} / \mathrm{L}$ ), and TG (median: $0.32 \mathrm{mmol} / \mathrm{L}$; $95 \%$ CI $0.20-0.43 \mathrm{mmol} / \mathrm{L}$ ) levels compared with statin therapy [181]. The therapeutic efficacy of EGb 716 as an antioxidant with free radical scavenging properties was explored in an RCT, in which the Abnormal Involuntary Movement Scale was used to quantify the severity of TD symptoms as the primary outcome. EGb $716(240 \mathrm{mg} /$ day $)$ outperformed the placebo group for minimizing the incidence of TD, and a meta-analysis suggested that adjunctive EGb 716 improved the incidence of TD among schizophrenia patients [133]. Patients with GAD (25-30 patients per group) treated with EGb 761(240 mg or $480 \mathrm{mg}$ placebo for 4 weeks) displayed increased cognitive abilities and reduced anxiety [136]. Flavonoids, lactones, kaempferol 3-O-?-D-glucopyranoside, isorhamnetin-3-O-glucoside, myricetin, ginkgolide $\mathrm{A}$, and bilobalide in $G$. biloba have displayed antiaging effects in human dermal fibroblasts (HDFs) through reduced ROS production and matrix metalloproteinase 1 (MMP-1) degradation. EGb tablets (19.2 mg per dose, 3 times a day) showed antidepressant effects in 136 older adult patients with depression through the modulation of serotonergic and dopaminergic neurotransmission [116] (Table 4).

\section{Conclusion and Future Perspectives}

G. biloba has been studied in a number of RCTs and has consistently demonstrated positive safety profiles and promising results for the treatment of a wide range of symptoms and diseases. Various tests have been conducted in both animal models and humans, and the underlying mechanisms of action have been defined. However, potential drug reactions and adverse effects are still being investigated in clinical trials. The uses of diverse methodologies, metrics, and research methods across studies have complicated the determination of effectiveness and the ability to perform cross-study comparisons. As a result, more sensitive and systematic outcome measures should be developed, in addition to the validation of interrater and intrarater reliabilities, concurrent test formats, test-retest reliability, and more accurate patient descriptions and diagnoses, to improve the ability to compare the outcomes of randomized studies. Future studies should discuss the fact that the doses used in many animal studies are not indicative of human dose levels. Reactions to G. biloba are influenced by various factors, including pharmacology, pharmacokinetics, pharmacodynamics, bioavailability, washout times, and long-term consequences of individual components. Dose-response properties, optimal pacing and length of clinical treatments, functions as an adjunctive therapy treatment, conditions for which $G$. biloba treatment is the most and least useful, side effects, and drug reactions have been excluded from the scope of this review; however, these factors are significant and deserve further research. Moreover, a wide variety of potential therapeutic effects for G. biloba such as protection of DNA from oxidative harm and mitochondrial dysfunction, the inhibition of mitochondrial ROS and apoptosis (mediated by mitochondria, caspases, and death receptors), function in the mitochondrial respiratory chain, mitochondrial DNA (mtDNA) effects, regulation of mitochondrial membrane potential, and intramitochondrial $\mathrm{Ca}^{2+}$ homeostasis have not yet been extensively studied.

In conclusion, $G$. biloba can serve as a natural substitute and complementary method for the treatment of a wide range of symptoms and disorders. When physicians and researchers are knowledgeable about the structure, indications, conditions, dosages/durations, pathways, concentration-dependent consequences and effectiveness, adverse reactions, and potential drug interactions, they can make decisions for improved patient experiences, safety, and analysis.

\section{Abbreviations}

G. biloba: Ginkgo biloba

AchE: Acetylcholinesterase

Arg-1: Arginase-1

Ach: Acetylcholine

ADL: Adenylosuccinate lyase

AFP: Alpha-fetoprotein

APE1: $\quad$ Apurinic/apyrimidinic endonuclease 1

ATL: Atlastin 1

BMI: $\quad$ Body mass index

BP: $\quad$ Blood pressure

BDNF: Brain-derived neurotrophic factor

ChAT: Choline acetyltransferase

COX: Cyclooxygenase

CXCL8: Chemokine (C-X-C motif) ligand 8

CD40L: Cluster of differentiation 40 ligand

CEA: Carcinoembryonic antigen

CBS: $\quad$ Cystathione beta synthase

CSE: Chromosome segregation

c-myc: $\quad$ Cellular myelocytomatosis

EGCG: Epigallocatechin-3-gallate

eNOS: $\quad$ Endothelial nitric oxide synthase

ERK1/2: Extracellular signal-regulated kinase1/2

FBG: $\quad$ Fasting blood glucose

FOXP1: $\quad$ Forkhead box protein P1

GSK3 $\beta$ : $\quad$ Glycogen synthase kinase-3-beta

8-OHdG: 8-Hydroxy-2'-deoxyguanosine

GRD: Glutathione reductase

GST: Glutathione-S-transferase

GB: Ginkgolide B

GM: Ginkgolides mixture

GPC-3: Glypican-3

GBEE: Ginkgo biloba exocarp extracts

GBE: $\quad$ Ginkgo biloba extract

GTL: Ginkgo terpene lactone

GC: Ginkgolide C

GSH: Glutathione

GPX1: Glutathione peroxidase 1

HCC: Hepatocellular carcinoma

HNE: Human neutrophil elastase

HbA1c: Glycohemoglobin 
HACU: High affinity choline uptake

HIF-1 $\alpha$ : Hypoxia inducible factor 1-alpha

HDL-C: High-density lipoprotein cholesterol

IFN- $\gamma$ : Interferon gamma

ING-3: $\quad$ Inhibitor of growth 3

IL: Interleukin

LDL-C: $\quad$ Low-density lipoprotein cholesterol

JNK: $\quad$-Jun N-terminal kinase

LPS: Lipopolysaccharide

MMP-1: $\quad$ Matrix metallo proteinase-1

MUC5AC: Mucin 5AC

MIC: $\quad$ Minimum inhibitory concentration

MDA: Malondialdehyde

NSSA: Nonenzymatic superoxide scavenger activity

n.m.: $\quad$ Not mentioned

NF-кB: $\quad$ Nuclear factor kappa light chain enhancer of activated B cells

OXPHOS: Oxidative phosphorylation

PP2A: $\quad$ Protein phosphatase 2

PPAR $\alpha$ Peroxisome proliferator-activated receptor

PXR: $\quad$ Pregnane $X$ receptor

PL: $\quad$ Pancreatic lipase

P38MAPK: p38 mitogen-activated protein kinase

p-KSR1/ Kinase suppressor of Ras 1

KSR1:

PF4: $\quad$ Platelet factor 4

p-Akt/Akt: Protein kinase B (PKB)

ROS: $\quad$ Reactive oxygen species

RNS: $\quad$ Reactive nitrogen species

STAT3: $\quad$ Signal transducer activator of transcription-3

Syk: $\quad$ Spleen tyrosine kinase

SOD: $\quad$ Superoxide dismutase

TG: $\quad$ Triglycerides

TC: Total count of WBC

TSSA: Total (enzymatic and nonenzymatic) superoxide scavenger activity

TNF: $\quad$ Tumor necrosis factor

VAI: Visceral adiposity index

VEGF: Vascular endothelial growth factor

VEGFR2: Vascular endothelial growth factor receptor 2

Wnt/ Wingless-related integration site beta-catechin

$\beta$-catenin:

XO: Xanthine oxidase.

\section{Data Availability}

The data used to support the findings of this study are included within the article.

\section{Conflicts of Interest}

The authors declare that they have no conflicts of interest.

\section{Authors' Contributions}

Noor-E-Tabassum and Rajib Das conceptualized the study, involved in data curation, developed methodology, and wrote original draft of the article. Mashia Subha Lami and Arka Jyoti Chakraborty visualized and investigated the study. Saikat Mitra visualized and investigated the study and performed formal analysis. Trina Ekawati Tallei, Rinaldi Idroes, Amany Abdel-Rahman Mohamed, Md. Jamal Hossain, and Kuldeep Dhama validated the study, performed formal analysis, and wrote and reviewed the article. Gomaa Mostafa-Hedeaband and Talha Bin Emran validated the study, performed formal analysis, wrote and reviewed the article, and supervised the study.

\section{Acknowledgments}

This work was funded by the Deanship of Scientific Research at Jouf University (DSR-2021-01-0373).

\section{References}

[1] L. F. B. Aacute Rbara, C. M. Michele, and B. L. d, F. Guilherme, "Ginkgo bilobaL.: phytochemical components and antioxidant activity," African Journal of Pharmacy and Pharmacology, vol. 9, 2015.

[2] T. K. Mohanta, A. Occhipinti, S. Atsbaha Zebelo, M. Foti, and C. M Bertea, "Ginkgo biloba responds to herbivory by activating early signaling and direct defenses," PLoS One, vol. 7, p. e32822, 2012.

[3] B. Singh, P. Kaur, Gopichand, R. D. Singh, and P. S. Ahuja, "Biology and chemistry of Ginkgo biloba," Fitoterapia, vol. 79, pp. 401-18, 2008.

[4] R. S. R. Tarun Belwal, L. Giri, B. Amit, M.. Tariq, and P. Kewlani: Ginkgo Biloba,” 2019.

[5] Q. Zhao, C. Gao, and Z. Cui, "Ginkgolide A reduces inflammatory response in high-glucose-stimulated human umbilical vein endothelial cells through STAT3-mediated pathway," International Immunopharmacology, vol. 25, pp. 242-8, 2015.

[6] Y. T. He, S. S. Xing, L. Gao, J. Wang, Q. C. Xing, and W. Zhang, "Ginkgo biloba attenuates oxidative DNA damage of human umbilical vein endothelial cells induced by intermittent high glucose," Die Pharmazie, vol. 69, pp. 203-7, 2014.

[7] S. B. Sarikçioğlu, G. Öner, and E. Tercan, “Antioxidant effect of EGb 761 on hydrogen peroxide-induced lipoperoxidation of G-6-PD deficient erythrocytes," Phytotherapy Research, vol. 18, 2004.

[8] A. A. Boligon, R. B. de Freitas, T. F. de Brum et al., "Phytochemical constituents and in vitro antioxidant capacity of Tabernaemontana catharinensis A. DC," Free Radicals and Antioxidants, vol. 3, no. 2, pp. 77-80, 2013.

[9] S. M. L. Vasconcelos, M. O. F. Goulart, and J. B. de F. Moura, "Espécies reativas de oxigênio e de nitrogênio, antioxidantes e marcadores de dano oxidativo em sangue humano: principais métodos analíticos para sua determinação," Química Nova, vol. 30, 2007.

[10] S. Wang, D. Wang, and Z. Liu, "Synergistic, additive and antagonistic effects of Potentilla fruticosa combined with EGb761 on antioxidant capacities and the possible mechanism," Industrial Crops and Products, vol. 67, 2015.

[11] P. C. Chan, Q. Xia, and P. P. Fu, "Ginkgo biloba leave extract: biological, medicinal, and toxicological effects," Journal of Environmental Science and Health-Part C: Environmental Carcinogenesis \& Ecotoxicology Reviews, vol. 25, 2007.

[12] B. Vellas, N. Coley, and P. J. Ousset, "Long-term use of standardised ginkgo biloba extract for the prevention of 
Alzheimer's disease (GuidAge): a randomised placebocontrolled trial," The Lancet Neurology, vol. 11, 2012.

[13] T. A. Van Beek, "Chemical analysis of Ginkgo biloba leaves and extracts," Journal of Chromatography A, vol. 967, no. 1, pp. 21-55, 2002.

[14] K. Nakanishi, "Terpene trilactones from gingko biloba: from ancient times to the 21st century," Bioorganic \& Medicinal Chemistry, vol. 13, pp. 4987-5000, 2005.

[15] S. Kressmann, A. Biber, M. Wonnemann, B. Schug, H. H. Blume, and W. E. Müller, "Influence of pharmaceutical quality on the bioavailability of active components from Ginkgo biloba preparations," Journal of Pharmacy and Pharmacology, vol. 54, no. 11, pp. 1507-1514, 2010.

[16] R. Akrami, M. H. Hashempur, and A. Tavakoli, "Effects of Fumaria parviflora $\mathrm{L}$ on uremic pruritus in hemodialysis patients: a randomized, double-blind, placebo-controlled trial," Jundishapur Journal of Natural Pharmaceutical Products, vol. 12, no. 3, 2017.

[17] A. M. Tareq, S. Farhad, A. B. M. Neshar Uddin et al., "Chemical profiles, pharmacological properties, and in silico studies provide new insights on Cycas pectinata," Heliyon, vol. 6, no. 6, Article ID e04061, 2020.

[18] M. H. Hashempur, M. S. Ghasemi, B. Daneshfard et al., "Efficacy of topical chamomile oil for mild and moderate carpal tunnel syndrome: a randomized double-blind placebo-controlled clinical trial," Complementary Therapies in Clinical Practice, vol. 26, pp. 61-67, 2017.

[19] A. Khiveh, M. H. Hashempur, M. Shakiba et al., "Effects of rhubarb ( Rheum ribes L.) syrup on dysenteric diarrhea in children: a randomized, double-blind, placebo-controlled trial," Journal of Integrative Medicine, vol. 15, no. 5, pp. 365-372, 2017.

[20] L. Liu, Y. Wang, J. Zhang, and S. Wang, "Advances in the chemical constituents and chemical analysis of Ginkgo biloba leaf, extract, and phytopharmaceuticals," Journal of Pharmacy Biomedicine Analytical, vol. 193, p. 2021.

[21] Biologically Active Secondary Metabolites from Ginkgo Biloba.

[22] W. Dong, S. Zhao, S. Wen et al., "A preclinical randomized controlled study of ischemia treated with Ginkgo biloba extracts: are complex components beneficial for treating acute stroke?" Current Research in Translational Medicine, vol. 68, no. 4, pp. 197-203, 2020.

[23] H. Ji, X. Zhou, W. Wei, W. Wu, and S. Yao, "Ginkgol Biloba extract as an adjunctive treatment for ischemic stroke," Medicine, vol. 99, no. 2, p. e18568, 2020.

[24] M. Z. Uddin, M. S. Rana, S. Hossain et al., "In vivo neuroprotective, antinociceptive, anti-inflammatory potential in Swiss albino mice and in vitro antioxidant and clot lysis activities of fractionated Holigarna longifolia Roxb. bark extract," Journal of Complementary and Integrative Medicine, vol. 17, no. 1, pp. 1-8, 2020.

[25] X. Wang, X. Gong, H. Zhang et al., "In vitro anti-aging activities of ginkgo biloba leaf extract and its chemical constituents," Food Science and Technology, vol. 40, no. 2, pp. 476-482, 2020.

[26] A. Hasler, G.-A. Gross, B. Meier, and O. Sticher, "Complex flavonol glycosides from the leaves of Ginkgo biloba," Phytochemistry, vol. 31, no. 4, pp. 1391-1394, 1992.

[27] Z. Al Mahmud, T. B. Emran, N. Qais, S. C. Bachar, M. Sarker, and M. Muhammad Nasir Uddin, "Evaluation of analgesic, anti-inflammatory, thrombolytic and hepatoprotective activities of roots of Premna esculenta (Roxb)," Journal of Basic and Clinical Physiology and Pharmacology, vol. 27, no. 1, pp. 63-70, 2016.

[28] H. L. Dong, S. Lin, and Q. L. Wu, "A new bilobalide isomer and two cis-coumaroylated flavonol glycosides from Ginkgo biloba leaves," Fitoterapia, vol. 142, p. 104516, 2020.

[29] P. Shu, M. Sun, J. Li, L. Zhang, and H. Xu, "Chemical constituents from Ginkgo biloba leaves and their cytotoxicity activity," Journal of Natural Medicines, vol. 74, pp. 269-274, 2020.

[30] T. A. van Beek and P. Montoro, "Chemical analysis and quality control of Ginkgo biloba leaves, extracts, and phytopharmaceuticals," Journal of Chromatography A, vol. 1216, no. 11, pp. 2002-2032, 2009.

[31] Y. Zhang, J. Liu, B. Yang, and Y. Zheng, "Ginkgo biloba extract inhibits astrocytic lipocalin-2 expression and alleviates neuroinflammatory injury via the JAK2/STAT3 pathway after ischemic brain stroke," Annual Meeting of the Japanese Pharmacological Society, vol. WCP2018, 2018.

[32] S. Yesmin, A. Paul, T. Naz et al., "Membrane stabilization as a mechanism of the anti-inflammatory activity of ethanolic root extract of Choi (Piper chaba)," Clinical Phytoscience, vol. 6, no. 1, pp. 1-10, 2020.

[33] S. jun Shan, J. Luo, and D. ran Xu, "Elucidation of micromolecular phenylpropanoid and lignan glycosides as the main antioxidants of Ginkgo seeds," Industrial Crops and Products, vol. 112, 2018.

[34] W. Li, Z. Luo, X. Liu et al., "Effect of Ginkgo biloba extract on experimental cardiac remodeling," BMC Complementary and Alternative Medicine, vol. 15, no. 1, p. 277, 2015

[35] Y. Wang, R. Wang, Y. Wang, R. Peng, Y. Wu, and Y. Yuan, " $\alpha$ Ginkgo biloba extract mitigates liver fibrosis and apoptosis by regulating p38 MAPK, NF- $\kappa \mathrm{B} / \mathrm{I} \kappa \mathrm{B} \alpha$, and $\mathrm{Bcl}-2 / \mathrm{Bax}$ signaling," Drug Design, Development and Therapy, vol. 9, pp. 6303-6317, 2015.

[36] Z. A. Okhti, M. E. Abdalah, and D. B. Hanna, "Phytochemical structure and Biological Effect of Ginkgo biloba leaves:A review," International Journal of Pharmacological Research, vol. 13, no. 2, 2021.

[37] M. Klein, K. Fiehe, and J. Gror, "Occurrence of neurotoxic 4'O-methylpyridoxine in ginkgo biloba leaves, ginkgo medications and Japanese ginkgo food," Planta Medica, vol. 62, no. 2, pp. 548-551, 1996.

[38] G. Tewari, B. Mohan, K. Kishor, L. M. Tewari, and T. K. Nailwal, "Volatile constituents of ginkgo biloba L. Leaves from Kumaun: a source of (E)-nerolidol and phytol," Journal of the Indian Chemical Society, vol. 93, 2015.

[39] D. Kaushik, V. Kumar, and H. Dureja, "Developments in nutraceuticals," Indian Journal of Pharmacology, vol. 35, no. 6, pp. 363-372, 2003.

[40] H. Y. Wang and Y. Q. Zhang, "The main active constituents and detoxification process of Ginkgo biloba seeds and their potential use in functional health foods," Journal of Food Composition and Analysis, vol. 83, 2019.

[41] K. A. Youdim and J. A. Joseph, "A possible emerging role of phytochemicals in improving age-related neurological dysfunctions: a multiplicity of effects," Free Radical Biology and Medicine, vol. 30, no. 6, pp. 583-594, 2001.

[42] K.-i. Hatano, T. Miyakawa, Y. Sawano, and M. Tanokura, "Antifungal and lipid transfer proteins from ginkgo (ginkgo biloba) seeds," Nuts and Seeds in Health and Disease Prevention, vol. 2011, pp. 527-534, 2011.

[43] N. Mei, X. Guo, Z. Ren, D. Kobayashi, K. Wada, and L. Guo, "Review of Ginkgo biloba-induced toxicity, from experimental studies to human case reports," Journal of 
Environmental Science and Health - Part C: Environmental Carcinogenesis \& Ecotoxicology Reviews, vol. 35, no. 1, pp. 1-28, 2017.

[44] H. Liu, M. Ye, and H. Guo, "An updated review of randomized clinical trials testing the improvement of cognitive function of ginkgo biloba extract in healthy people and alzheimer's patients," Frontiers in Pharmacology, vol. 10, 2020.

[45] E. Pereira, L. Barros, and I. C. F. R. Ferreira, "Chemical characterization of Ginkgo biloba L. and antioxidant properties of its extracts and dietary supplements," Industrial Crops and Products, vol. 51, pp. 244-248, 2013.

[46] R. Tao, C. Wang, J. Ye, H. Zhou, and H. Chen, "Polyprenols of ginkgo biloba enhance antibacterial activity of five classes of antibiotics," BioMed Research International, vol. 2016, p. 4191938, 2016.

[47] T. A. Bristy, N. Barua, A. Montakim Tareq et al., "Deciphering the pharmacological properties of methanol extract of Psychotria calocarpa leaves by in vivo, in vitro and in silico approaches," Pharmaceuticals, vol. 13, no. 8, p. 183, 2020.

[48] S. H. Baek, J. H. Lee, C. Kim, J. H. Ko, S. H. Ryu, and S. G. Lee, "Ginkgolic acid C 17:1, derived from Ginkgo biloba leaves, suppresses constitutive and inducible STAT3 activation through induction of PTEN and SHP-1 tyrosine phosphatase," Molecules, vol. 22, 2017.

[49] M. Li, B. Li, and Z. M. Xia, "Anticancer effects of five biflavonoids from ginkgo biloba 1 . Male flowers in vitro," Molecules, vol. 24, no. 8, 2019.

[50] In Silico Screening Ginkgolide Compounds from Ginkgo Biloba Extract Injection to Affect Drug-Metabolzing Enzymes.

[51] S. Nishimon, M. Yamaguchi, H. Muraki, N. Sakai, and S. Nishino, "Intraperitoneal injection of ginkgolide B, a major active compound of Ginkgo biloba, dose-dependently increases the amount of wake and decreases non-rapid eye movement sleep in C57BL/6 mice," Neuroscience Letters, vol. 722, p. 134832, 2020.

[52] A. M. Silva, S. C. Silva, J. P. Soares et al., "Ginkgo biloba L. Leaf extract protects HepG2 cells against paraquat-induced oxidative DNA damage," Plants, vol. 8, no. 12, 2019.

[53] P. Chang, Y. Xu, D. Y. Zhou, J. Di Wu, and S. L. Ma, "Effects of polysaccharides from Ginkgo biloba on proliferation of 4T1 breast cancer cells and expression of GLUT family genes," Chinese Pharmacological Bulletin, vol. 34, 2018.

[54] Y. Lu, F. Jiang, H. Jiang et al., "Gallic acid suppresses cell viability, proliferation, invasion and angiogenesis in human glioma cells," European Journal of Pharmacology, vol. 641, pp. 102-7, 2010.

[55] M. Kaur, B. Velmurugan, S. Rajamanickam, R. Agarwal, and C. Agarwal, "Gallic acid, an active constituent of grape seed extract, exhibits anti-proliferative, pro-apoptotic and antitumorigenic effects against prostate carcinoma xenograft growth in nude mice," Pharmaceutical Research, vol. 26, pp. 2133-40, 2009.

[56] R. Varì, M. D’Archivio, C. Filesi et al., "Protocatechuic acid induces antioxidant/detoxifying enzyme expression through JNK-mediated Nrf2 activation in murine macrophages," The Journal of Nutritional Biochemistry, vol. 22, pp. 409-17, 2011.

[57] E. C. Yip, A. S. Chan, H. Pang, Y. K. Tam, and Y. H. Wong, "Protocatechuic acid induces cell death in HepG2 hepatocellular carcinoma cells through a c-Jun N-terminal kinasedependent mechanism," Cell Biology and Toxicology, vol. 22, pp. 293-302, 2006.

[58] Z. Yan, R. Fan, S. Yin et al., "Protective effects of Ginkgo biloba leaf polysaccharide on nonalcoholic fatty liver disease and its mechanisms," International Journal of Biological Macromolecules, vol. 80, pp. 573-80, 2015.

[59] F. Eisvand, B. M. Razavi, and H. Hosseinzadeh, "The effects of Ginkgo biloba on metabolic syndrome: a review," Phytotherapy Research, vol. 34, no. 8, pp. 1798-1811, 2020.

[60] S. Mahadevan and Y. Park, "Multifaceted therapeutic benefits of Ginkgo biloba L.: chemistry, efficacy, safety, and uses," Journal of Food Science, vol. 73, pp. R14-9, 2008.

[61] Y. Sasaki, T. Noguchi, E. Yamamoto et al., "Effects of Ginkgo biloba extract (EGb 761) on cerebral thrombosis and blood pressure in stroke-prone spontaneously hypertensive rats," Clinical and Experimental Pharmacology and Physiology, vol. 29, pp. 963-7, 2002.

[62] O. Napryeyenko and I. Borzenko, "Ginkgo biloba special extract in dementia with neuropsychiatric features," Arzneimittelforschung, vol. 57, 2011.

[63] L. H. Fan, K. Z. Wang, and B. Cheng, "Effects of Ginkgo biloba extract on lipid peroxidation and apoptosis after spinal cord ischemia/reperfusion in rabbits," Chinese journal of traumatology=Zhonghua chuang shang za zhi, vol. 9, pp. 77-81, 2006.

[64] S. Akiba, M. Chiba, Y. Mukaida, A. Tamura, and T. Sato, "The leaf extract of Ginkgo Biloba L. suppresses oxidized LDL-stimulated fibronectin production through an antioxidant action in rat mesangial cells," British Journal of Pharmacology, vol. 142, pp. 419-24, 2004.

[65] A. Mashayekh, D. L. Pham, D. M. Yousem, M. Dizon, P. B. Barker, and D. D. Lin, "Effects of Ginkgo biloba on cerebral blood flow assessed by quantitative MR perfusion imaging: a pilot study," Neuroradiology, vol. 53, pp. 185-91, 2011.

[66] K. Shinozuka, K. Umegaki, Y. Kubota et al., "Feeding of Ginkgo biloba extract (GBE) enhances gene expression of hepatic cytochrome P-450 and attenuates the hypotensive effect of nicardipine in rats," Life Sciences, vol. 70, pp. 2783-92, 2002.

[67] S. R. Naik and V. S. Panda, "Antioxidant and hepatoprotective effects of Ginkgo biloba phytosomes in carbon tetrachloride-induced liver injury in rodents," Liver International: Official Journal of the International Association for the Study of the Liver, vol. 27, p. 393, 2007.

[68] K. S. Cho, I. M. Lee, and S. Sim, "Ginkgo biloba extract (EGb $\left.761^{\circledR}\right)$ inhibits glutamate-induced up-regulation of tissue plasminogen activator through inhibition of c-fos translocation in rat primary cortical neurons," Phytotherapy Research, vol. 30, 2016.

[69] T. K. Mohanta, Y. Tamboli, and P. K. Zubaidha, "Phytochemical and medicinal importance ofGinkgo bilobaL," Natural Product Research, vol. 28, no. 10, pp. 746-752, 2014.

[70] F. Wu, W. Shi, G. Zhou et al., "Ginkgolide B functions as a determinant constituent of Ginkgolides in alleviating lipopolysaccharide-induced lung injury," Biomedicine \& pharmacotherapy = Biomedecine \& pharmacotherapie, vol. 81, pp. 71-78, 2016.

[71] Z. Tao, W. Jin, M. Ao, S. Zhai, H. Xu, and L. Yu, "Evaluation of the anti-inflammatory properties of the active constituents in Ginkgo biloba for the treatment of pulmonary diseases," Food \& Function, vol. 10, pp. 2209-2220, 2019.

[72] H. H. Ahmed, W. G. Shousha, H. A. El-Mezayen, S. A. ElToumy, A. H. Sayed, and A. R. Ramadan, "Biochemical and molecular evidences for the antitumor potential of Ginkgo biloba leaves extract in rodents," Acta Biochimica Polonica, vol. 64, pp. 25-33, 2017. 
[73] D. Han, C. Cao, Y. Su et al., “ $\beta$ Ginkgo biloba exocarp extracts inhibits angiogenesis and its effects on Wnt/ $\beta$-catenin-VEGF signaling pathway in Lewis lung cancer," Journal of Ethnopharmacology, vol. 192, pp. 406-412, 2016.

[74] Y. Qian, L. Xia, W. Shi, J. J. Sun, and Y. Q. Sun, "The effect of EGB on proliferation of gastric carcinoma SGC7901 cells," Clinical and Translational Oncology, vol. 18, 2016.

[75] K. M. Nash and Z. A. Shah, "Current perspectives on the beneficial role of Ginkgo biloba in neurological and cerebrovascular disorders," Integrative Medicine Insights, vol. 10, pp. 1-9, 2015.

[76] D. R. Whiting, L. Guariguata, C. Weil, and J. Shaw, "IDF Diabetes Atlas: global estimates of the prevalence of diabetes for 2011 and 2030," Diabetes Research and Clinical Practice, vol. 94, pp. 311-21, 2011.

[77] A. Priyanka, G. Sindhu, G. L. Shyni, M. R. Preetha Rani, V. M. Nisha, and K. G. Raghu, "Bilobalide abates inflammation, insulin resistance and secretion of angiogenic factors induced by hypoxia in 3T3-L1 adipocytes by controlling NF$\kappa \mathrm{B}$ and JNK activation," International Immunopharmacology, vol. 42, pp. 209-217, 2017.

[78] B. K. Hirata, R. M. Banin, A. P. Dornellas et al., "Ginkgo biloba extract improves insulin signaling and attenuates inflammation in retroperitoneal adipose tissue depot of obese rats," Mediators of Inflammation, vol. 2015, p. 419106, 2015.

[79] P. K. Liu, Z. M. Weng, G. B. Ge, and H. L. Li, "Biflavones from Ginkgo biloba as novel pancreatic lipase inhibitors: inhibition potentials and mechanism," International Journal of Biological Macromolecules, vol. 118, pp. 2216-2223, 2018.

[80] L. Luo, Y. Li, and D. Wang, "Ginkgolide B lowers body weight and ameliorates hepatic steatosis in high-fat dietinduced obese mice correlated with pregnane $\mathrm{X}$ receptor activation," RSC Advances, vol. 60, 2017.

[81] W. C. Huang, Y. L. Chen, H. C. Liu, S. J. Wu, and C. J. Liou, "Ginkgolide $\mathrm{C}$ reduced oleic acid-induced lipid accumulation in HepG2 cells," Saudi Pharmaceutical Journal, vol. 26, 2018.

[82] H. Kang, "Hypocholesterolemic effect of ginkgo biloba seeds extract from high fat diet mice," Biomedical Science Letters, vol. 23, 2017.

[83] H. Liu, X. Pei, and K. Shi, "Effects of replacing wheat flour with detoxified ginkgo nut powder on lipid metabolism of obese C57Bl/6J male mice," Food and Agricultural Immunology, vol. 29, 2018.

[84] I. Afanas'ev, "Mechanisms of superoxide signaling in epigenetic processes: relation to aging and cancer," Aging and Disease, vol. 6, 2015.

[85] F. Bonomini, L. F. Rodella, and R. Rezzani, "Metabolic syndrome, aging and involvement of oxidative stress," Aging and Disease, vol. 6, pp. 109-20, 2015.

[86] N. Kaur, M. Dhiman, J. R. Perez-Polo, and A. K. Mantha, " $\beta$ Ginkgolide $\mathrm{B}$ revamps neuroprotective role of apurinic/ apyrimidinic endonuclease 1 and mitochondrial oxidative phosphorylation against $\mathrm{A} \beta 25-35$-induced neurotoxicity in human neuroblastoma cells," Journal of Neuroscience Research, vol. 93, pp. 938-47, 2015.

[87] X. Zhou, Y. Qi, and T. Chen, "Long-term pre-treatment of antioxidant Ginkgo biloba extract EGb-761 attenuates cerebral-ischemia-induced neuronal damage in aged mice," Biomedicine \& pharmacotherapy = Biomedecine \& pharmacotherapie, vol. 85, pp. 256-263, 2017.

[88] N. Pazyar, R. Yaghoobi, E. Rafiee, A. Mehrabian, and A. Feily, "Skin wound healing and phytomedicine: a review," Skin Pharmacology and Physiology, vol. 27, pp. 303-10, 2014.
[89] S. Okumus, S. Taysi, and M. Orkmez, "The effects of oral Ginkgo biloba supplementation on radiation-induced oxidative injury in the lens of rat," Pharmacognosy Magazine, vol. 7, 2011.

[90] S. Trompezinski, M. Bonneville, and I. Pernet, "Gingko biloba extract reduces VEGF and CXCL-8/IL-8 levels in keratinocytes with cumulative effect with epigallocatechin-3gallate," Archives of Dermatological Research, vol. 302, pp. 183-9, 2010.

[91] C. Dong, "Protective effect of extract of ginkgo biloba 761 against frostbite injury in rats," Plastic and Reconstructive Surgery, vol. 143, 2020.

[92] S. Q. Liu, C. Y. Xu, M. B. Qin, and L. Tan, "Ginkgo biloba extract enhances chemotherapy sensitivity and reverses chemoresistance through suppression of the KSR1-mediated ERK1/2 pathway in gastric cancer cells," Oncology Reports, vol. 33, pp. 2871-82, 2015.

[93] Study on Isolation, Identification and the Antibacterial Activity of Ginkgolic Acids from Ginkgo Biloba Seeds.

[94] Ginkgo Biloba Extract on Brain Oxidative Condition after Cisplatin Exposure.

[95] K. J. Kwon, E. J. Lee, K. S. Cho, D. H Cho, C. Y Shin, and S. H Han, " $\beta$ Ginkgo biloba extract (Egb761) attenuates zincinduced tau phosphorylation at Ser262 by regulating GSK3 $\beta$ activity in rat primary cortical neurons," Food \& Function, vol. 6, pp. 2058-2067, 2015.

[96] M. Belviranli and N. Okudan, "The effects of Ginkgo biloba extract on cognitive functions in aged female rats: the role of oxidative stress and brain-derived neurotrophic factor," Behavioural Brain Research, vol. 278, 2015.

[97] X. Liu, Y. Yan, L. Bao, B. Chen, Y. Zhao, and R. Qi, "Ginkgolide B inhibits platelet release by blocking Syk and p38 MAPK phosphorylation in thrombin-stimulated platelets," Thrombosis Research, vol. 134, pp. 1066-73, 2014.

[98] P. Huang, L. Zhang, C. Chai, and X. C. Qian, "Effects of food and gender on the pharmacokinetics of ginkgolides A, B, C and bilobalide in rats after oral dosing with ginkgo terpene lactones extract," Journal of Pharmaceutical and Biomedical Analysis, vol. 100, pp. 138-144, 2014.

[99] A. O. Abdel-Zaher, H. S. M. Farghaly, A. E. M. El-Refaiy, and A. M. Abd-Eldayem, "Protective effect of the standardized extract of ginkgo biloba (EGb761) against hypertension with hypercholesterolemia-induced renal injury in rats: insights in the underlying mechanisms," Biomedicine \& Pharmacotherapy, vol. 95, 2017.

[100] W. Wan, C. Zhang, M. Danielsen et al., "EGb761 improves cognitive function and regulates inflammatory responses in the APP/PS1 mouse," Experimental Gerontology, vol. 81, pp. 92-100, 2016.

[101] S. Christoph, H. Julia, L. Helene, and K. Jochen, "Effects of ginkgo biloba extract EGb 761, donepezil and their combination on central cholinergic function in aged rats," Journal of Pharmacy and Pharmaceutical Sciences, vol. 18, 2015.

[102] S. Hajirezaee, A. Rafieepour, S. Shafiei, and R. Rahimi, "Immunostimulating effects of Ginkgo biloba extract against toxicity induced by organophosphate pesticide, diazinon in rainbow trout, Oncorhynchus mykiss: innate immunity components and immune-related genes," Environmental Science and Pollution Research, vol. 26, no. 9, pp. 8798-8807, 2019.

[103] M. Li, B. Li, Y. Hou et al., "Anti-inflammatory effects of chemical components from Ginkgo biloba L. male flowers on lipopolysaccharide-stimulated RAW264.7 macrophages," Phytotherapy Research, vol. 33, no. 4, pp. 989-997, 2019. 
[104] L. T. Al Kury, F. Dayyan, and F. Ali Shah, "Ginkgo biloba extract protects against methotrexate-induced hepatotoxicity: a computational and pharmacological approach," Molecules, vol. 25, no. 11, 2020.

[105] Q. Hu, P. Shen, S. Bai et al., "Metabolite-related antidepressant action of diterpene ginkgolides in the prefrontal cortex," Neuropsychiatric Disease and Treatment, vol. 14, pp. 999-1011, 2018.

[106] M. M. Rahaman, A. Rakib, and S. Mitra, "The genus curcuma and inflammation: overview of the pharmacological perspectives," Plants, vol. 10, no. 1, pp. 1-19, 2021.

[107] J. Vane and R. Botting, "Inflammation and the mechanism of action of anti-inflammatory drugs," The FASEB Journal, vol. 1, no. 2, pp. 89-96, 1987.

[108] E. G. Porcelli, "Chronic inflammation," The Journal of the American Dental Association, vol. 149, no. 9, pp. 750-751, 2018.

[109] S. J. Maleki, J. F. Crespo, and B. Cabanillas, "Anti-inflammatory effects of flavonoids," Food Chemistry, vol. 299, p. 125124, 2019.

[110] K. Ganesan, M. Jayachandran, and B. Xu, "A critical review on hepatoprotective effects of bioactive food components," Critical Reviews in Food Science and Nutrition, vol. 58, no. 7, pp. 1165-1229, 2018.

[111] M.-Z. Weng, X.-P. Zhou, J.-G. Jia et al., "The hepatic protective mechanism of Ginkgo biloba extract in rats with obstructive jaundice," Bosnian Journal of Basic Medical Sciences, vol. 11, no. 4, pp. 209-213, 2011.

[112] R. Chávez-Morales, F. Jaramillo-Juárez, F. Posadas del Río, M. Reyes-Romero, M. Rodríguez-Vázquez, and M. Martínez-Saldaña, "Protective effect of Ginkgo biloba extract on liver damage by a single dose of $\mathrm{CCl} 4$ in male rats," Human \& Experimental Toxicology, vol. 30, no. 3, pp. 209-216, 2011.

[113] K. Ashok Shenoy, S. N. Somayaji, and K. L. Bairy, "Hepatoprotective effects of Ginkgo biloba against carbon tetrachloride induced hepatic injury in rats," Indian Journal of Pharmacology, vol. 33, no. 4, pp. 260-266, 2001.

[114] Z. Y. Zhou, S. Q. Tang, Y. M. Zhou, H. S. Luo, and X. Liu, "Antioxidant and hepatoprotective effects of extract of ginkgo biloba in rats of non-alcoholic steatohepatitis," Saudi Medical Journal, vol. 31, no. 10, pp. 1114-1118, 2010.

[115] E. Vaghef-Mehrabany, V. Maleki, M. Behrooz, F. Ranjbar, and M. Ebrahimi-Mameghani, "Can psychobiotics "mood" ify gut? An update systematic review of randomized controlled trials in healthy and clinical subjects, on anti-depressant effects of probiotics, prebiotics, and synbiotics," Clinical Nutrition, vol. 39, no. 5, pp. 1395-1410, 2020.

[116] C. X. Dai, C. C. Hu, Y. S. Shang, and J. Xie, "Role of Ginkgo biloba extract as an adjunctive treatment of elderly patients with depression and on the expression of serum S100B," Medicine, vol. 97, no. 39, p. e12421, 2018.

[117] P. Chen, M. Hei, L. Kong et al., "One water-soluble polysaccharide from Ginkgo biloba leaves with antidepressant activities via modulation of the gut microbiome," Food \& Function, vol. 10, no. 12, pp. 8161-8171, 2019.

[118] Z.-H. Liang, Y.-B. Jia, M.-L. Wang et al., "Efficacy of ginkgo biloba extract as augmentation of venlafaxine in treating post-stroke depression," Neuropsychiatric Disease and Treatment, vol. 15, pp. 2551-2557, 2019.

[119] T. Wang, S. Bai, W. Wang et al., "Diterpene ginkgolides exert an antidepressant effect through the NT3-trkA and rasMAPK pathways," Drug Design, Development and Therapy, vol. 14, pp. 1279-1294, 2020.
[120] L. Zhang, J. Liu, Y. Ge, and M. Liu, "Ginkgo biloba extract reduces Hippocampus inflammatory responses, improves cardiac functions and depressive behaviors in A heart failure mouse model," Neuropsychiatric Disease and Treatment, vol. 15, pp. 3041-3050, 2019.

[121] S. A. Ayatollahi, S. Khoshsirat, S. Khoshsirat et al., "Ginkgo biloba modulates hippocampal BDNF expression in a rat model of chronic restraint stress-induced depression," Physiology and Pharmacology, vol. 24, no. 4, pp. 285-297, 2020.

[122] T. B. Emran, M. M. N. Uddin, A. Rahman, Z. Uddin, and M. Islam, "Phytochemical, antimicrobial, cytotoxic, analgesic and anti-inflammatory properties of Azadirachta indica: a therapeutic study," Journal of Bioanalysis and Biomedicine, vol. 12, no. 2, p. 7, 2015.

[123] S. E. Dal Belo, L. R. Gaspar, P. M. B. G. Maia Campos, and J.-P. Marty, "Skin penetration of epigallocatechin-3-gallate and quercetin from green tea and Ginkgo biloba extracts vehiculated in cosmetic formulations," Skin Pharmacology and Physiology, vol. 22, no. 6, pp. 299-304, 2009.

[124] C. Shi, S. Xiao, J. Liu et al., "Ginkgo bilobaextract EGb761 protects against aging-associated mitochondrial dysfunction in platelets and hippocampi of SAMP8 mice," Platelets, vol. 21, no. 5, pp. 373-379, 2010.

[125] A. Rakib, S. Ahmed, M. A. Islam et al., "Pharmacological studies on the antinociceptive, anxiolytic and antidepressant activity of Tinospora crispa," Phytotherapy Research, vol. 34, no. 11, pp. 2978-2984, 2020.

[126] Y. Liu, Y. Liu, and W. Zhang, "Network pharmacology-based strategy to investigate the pharmacological mechanisms of ginkgo biloba extract for aging," Evidence-Based Complement. Alternative Medicine, vol. 2020, Article ID 8508491, 10 pages, 2020.

[127] D. Kajaria, J. Tripathi, S. Tiwari, and B. Pandey, "Immunomodulatory effect of ethanolic extract of Shirishadi compound," AYU (An International Quarterly Journal of Research in Ayurveda), vol. 34, no. 3, p. 322, 2013.

[128] Q. Ren, J. Chen, Y. Ding et al., "In vitro antioxidant and immunostimulating activities of polysaccharides from Ginkgo biloba leaves," International Journal of Biological Macromolecules, vol. 124, pp. 972-980, 2019.

[129] A. H. Xu, L. Ren, Y. Y. Zheng, and H. S. Chen, "Immunomodulatory effect of Ginkgo biloba exocarp polysaccharides on immunosuppressive mice induced by cyclophosphamide," Chinese Journal of Pharmacology and Toxicology, vol. 22, no. 1, pp. 69-72, 2008.

[130] D. Parsad, R. Pandhi, and A. Juneja, "Effectiveness of oral Ginkgo biloba in treating limited, slowly spreading vitiligo," Clinical and Experimental Dermatology, vol. 28, no. 3, pp. 285-287, 2003.

[131] J. Shams, S. S. P. Gudarzi, and A.-R. Norouzi, “The efficacy and safety of add-on ginko TD (ginkgo ) treatment for PTSD: results of a 12-week double-blind placebo-controlled study," Iranian Journal of Psychiatry and Behavioral Sciences, vol. 2, pp. 58-64, 2007.

[132] X. Y. Zhang, W.-F. Zhang, D.-F. Zhou et al., "Brain-derived neurotrophic factor levels and its val66met gene polymorphism predict tardive dyskinesia treatment response to ginkgo biloba," Biological Psychiatry, vol. 72, no. 8, pp. 700-706, 2012.

[133] W. Zheng, Y. Q. Xiang, C. H. Ng, G. S Ungvari, H. F Chiu, and Y. T Xiang, "Extract of ginkgo biloba for tardive dyskinesia: meta-analysis of randomized controlled trials," Pharmacopsychiatry, vol. 49, no. 3, pp. 107-111, 2016. 
[134] W. F. Zhang, Y. L. Tan, X. Y. Zhang, R. C. Chan, H. R. Wu, and D. F. Zhou, "Extract of Ginkgo biloba treatment for tardive dyskinesia in schizophrenia: a randomized, doubleblind, placebo-controlled trial," Journal of Clinical Psychiatry, vol. 72, no. 5, pp. 615-621, 2011.

[135] D. W. Phillips and J. Diebler, "Generalized anxiety disorder (GAD)," Encyclopedia of Victimology and Crime Prevention, 2012.

[136] S. Singh, G. Barreto, G. Aliev, and V. Echeverria, "Ginkgo biloba as an alternative medicine in the treatment of anxiety in dementia and other psychiatric disorders," Current Drug Metabolism, vol. 18, no. 2, pp. 112-119, 2016.

[137] V. Carola, F. D'Olimpio, E. Brunamonti, F. Mangia, and P. Renzi, "Evaluation of the elevated plus-maze and openfield tests for the assessment of anxiety-related behaviour in inbred mice," Behavioural Brain Research, vol. 134, no. 1-2, pp. 49-57, 2002.

[138] I. Baitharu, S. N. Deep, V. Jain et al., "Corticosterone synthesis inhibitor metyrapone ameliorates chronic hypobaric hypoxia induced memory impairment in rat," Behavioural Brain Research, vol. 228, no. 1, pp. 53-65, 2012.

[139] H. Woelk, K. H. Arnoldt, M. Kieser, and R. Hoerr, "Ginkgo biloba special extract EGb 761 in generalized anxiety disorder and adjustment disorder with anxious mood: a randomized, double-blind, placebo-controlled trial," Journal of Psychiatric Research, vol. 41, no. 6, pp. 472-480, 2007.

[140] P. Montes, E. Ruiz-Sanchez, C. Rojas, and P. Rojas, "Ginkgo biloba extract 761: a review of basic studies and potential clinical use in psychiatric disorders," CNS \& Neurological Disorders-Drug Targets, vol. 14, no. 1, pp. 132-149, 2015.

[141] J. Nordqvist, "Health benefits of Gingko biloba," Medical news and health news, vol. 2017, pp. 1-10, 2017.

[142] Hypertension. A Global Perspective.

[143] E. M. Konta, M. R. Almeida, C. L. Do Amaral et al., "Evaluation of the antihypertensive properties of yellow passion fruit pulp (passiflora edulis sims f. flavicarpa deg.) in spontaneously hypertensive rats," Phytotherapy Research: PT, vol. 28, pp. 28-32, 2014.

[144] W. Zuo, F. Yan, B. Zhang, J. Li, and D. Mei, "Advances in the studies of Ginkgo biloba leaves extract on aging-related diseases," Aging and disease, vol. 8, no. 6, pp. 812-826, 2017.

[145] X. Castellon and V. Bogdanova, "Chronic inflammatory diseases and endothelial dysfunction," Aging and Disease, vol. 25, 2016.

[146] J. Budni, T. Bellettini-Santos, F. Mina, M. L. Garcez, and A. I. Zugno, "The involvement of BDNF, NGF and GDNF in aging and Alzheimer's disease," Aging and Disease, vol. 6, pp. 331-41, 2015.

[147] T. Isah, "Rethinking Ginkgo biloba L.: medicinal uses and conservation," Pharmacognosy Reviews, vol. 9, no. 18, pp. 140-148, 2015.

[148] H. R. Lieberman, M. D. Kellogg, V. L. Fulgoni, and S. Agarwal, "Moderate doses of commercial preparations of Ginkgo biloba do not alter markers of liver function but moderate alcohol intake does: a new approach to identify and quantify biomarkers of "adverse effects" of dietary supplements," Regulatory Toxicology and Pharmacology, vol. 84, pp. 45-53, 2017.

[149] B. Jiang, H. Chen, H. Zhao, W. Wu, and Y. Jin, "Structural features and antioxidant behavior of lignins successively extracted from ginkgo shells (Ginkgo biloba L), International Journal of Biological Macromolecules, vol. 163, pp. 694-701, 2020.
[150] S. K. Kushwaha, C. S. Sharma, H. P. Singh, A. Ankalgi, and M. S. Ranawat, Ginkgo Biloba a Source of Bioactive Natural Products: A Review, 2014.

[151] D. Jain, S. Pancholi, and R. Patel, "Synergistic antioxidant activity of green tea with some herbs," "Journal of Advanced Pharmaceutical Technology \& Research, vol. 2, no. 3, p. 177, 2011.

[152] X. Zhu, Z. Li, C. Li, J. Zhang, Z. Zou, and J. Wang, “Ginkgo biloba extract and Aspirin synergistically attenuate activated platelet-induced ROS production and LOX-1 expression in human coronary artery endothelial cells," Phytomedicine, vol. 20, no. 2, pp. 114-119, 2013.

[153] S. Akiba, T. Kawauchi, T. Oka, T. Hashizume, and T. Sato, "Inhibitory effect of the leaf extract of Ginkgo biloba L. on oxidative stress-induced platelet aggregation," IUBMB Life, vol. 46, no. 6, pp. 1243-1248, 1998.

[154] R. Tao, C.-Z. Wang, and Z.-W. Kong, "Antibacterial/antifungal activity and synergistic interactions between polyprenols and other lipids isolated from Ginkgo Biloba L. leaves," Molecules, vol. 18, no. 2, pp. 2166-2182, 2013.

[155] B. J. Diamond and M. R. Bailey, "Ginkgo biloba: indications, mechanisms, and safety," Psychiatric Clinics of North America, vol. 36, pp. 73-83, 2013.

[156] Z. H. Liu, D. M. Wang, S. F. Fan, D. W. Li, and Z. W. Luo, "Synergistic effects and related bioactive mechanism of Potentilla fruticosa L. leaves combined with Ginkgo biloba extracts studied with microbial test system (MTS)," BMC Complementary and Alternative Medicine, vol. 16, no. 1, p. 495, 2016.

[157] P. M. Campos, M. D. Gianeti, D. G. Mercurio, and L. R. Gaspar, "Synergistic effects of green tea and Ginkgo biloba extracts on the improvement of skin barrier function and elasticity," Journal of Drugs in Dermatology: Journal of Drugs in Dermatology, vol. 13, no. 9, pp. 1092-1097, 2014.

[158] C. Di Lorenzo, A. Ceschi, and F. Colombo, "Identification and quantification of biomarkers to confirm the poisoning by Ginkgo biloba seeds in a 2-year-old boy," Toxicology Research, vol. 4, 2015.

[159] D. Kobayashi, T. Yoshimura, A. Johno, K. Sasaki, and K. Wada, "Toxicity of 4'-O-methylpyridoxine-5' -glucoside in Ginkgo biloba seeds," Food Chemistry, vol. 126, 2011.

[160] C. Di Lorenzo, A. Ceschi, and H. Kupferschmidt, "Adverse effects of plant food supplements and botanical preparations: a systematic review with critical evaluation of causality," British Journal of Clinical Pharmacology, vol. 79, 2015.

[161] H.-s. Jang, S. Y. Roh, E. H. Jeong, B.-S. Kim, and M. K. Sunwoo, "Ginkgotoxin induced seizure caused by vitamin B6 deficiency," Journal of Epilepsy Research, vol. 5, no. 2, pp. 104-106, 2015.

[162] E. S. Fernandes, R. M. Pinto, J. E. de Paula Reis, M. de Oliveira Guerra, and V. M. Peters, "Effects of Ginkgo biloba extract on the embryo-fetal development in wistar rats," Birth Defects Research. Part B, Developmental and Reproductive Toxicology, vol. 89, pp. 133-138, 2010.

[163] A. J. Kellermann and C. Kloft, "Is there a risk of bleeding associated with standardized Ginkgo biloba extract therapy? A systematic review and meta-analysis," Pharmacotherapy: The Journal of Human Pharmacology and Drug Therapy, vol. 31, 2011.

[164] P. V. Carlile, "Unexplained alveolar hemorrhage associated with ginkgo and ginseng use," Journal of bronchology \& interventional pulmonology, vol. 22, pp. 170-2, 2015.

[165] J. L. Pedroso, C. C. Henriques Aquino, M. L. Escórcio Bezerra et al., "Ginkgo biloba and cerebral bleeding: a case 
report and critical review," The Neurologist, vol. 17, pp. 89-90, 2011.

[166] G. J. Stoddard, M. Archer, L. Shane-McWhorter et al., "Ginkgo and warfarin interaction in a large veterans administration population," AMIA Annual Symposium proceedings. AMIA Symposium, vol. 2015, pp. 1174-83, 2015.

[167] E. Hotta, R. Tamagawa-Mineoka, and N. Katoh, "Allergic contact dermatitis due to ginkgo tree fruit and leaf," European Journal of Dermatology: EJD, vol. 23, pp. 548-9, 2013.

[168] V. Russo, A. Rago, G. M. Russo, R. Calabrò, and G. Nigro, "Ginkgo biloba: an ancient tree with new arrhythmic side effects," Journal of Postgraduate Medicine, vol. 57, p. 221, 2011.

[169] M. Luijten, E. D. Olthof, and B. C. Hakkert, "An integrative test strategy for cancer hazard identification," Critical Reviews in Toxicology, vol. 46, 2016.

[170] M. J. Hoenerhoff, A. R. Pandiri, and S. A. Snyder, "Hepatocellular carcinomas in $\mathrm{B} 6 \mathrm{C} 3 \mathrm{~F} 1$ mice treated with ginkgo biloba extract for two years differ from spontaneous liver tumors in cancer gene mutations and genomic pathways," Toxicologic Pathology, vol. 41, 2013.

[171] L. Guo, N. Mei, W. Liao, P. C. Chan, and P. P. Fu, "Ginkgo biloba extract induces gene expression changes in xenobiotics metabolism and the myc-centered network," OMICS: A Journal of Integrative Biology, vol. 14, pp. 75-90, 2010.

[172] U. Zehra, M. Tahir, and K. P. Lone, "Ginkgo biloba induced malformations in mice," Journal of the College of Physicians and Surgeons--Pakistan: JCPSP, vol. 20, pp. 117-21, 2010.

[173] E. Koch, M. Nöldner, and J. Leuschner, "Reproductive and developmental toxicity of the Ginkgo biloba special extract EGb $761^{\circledR}$ in mice," Phytomedicine, vol. 21, 2013.

[174] H. Lin, X. Guo, S. Zhang et al., "Mechanistic evaluation of ginkgo biloba leaf extract-induced genotoxicity in L5178Y cells," Toxicological Sciences, vol. 139, no. 2, pp. 338-349, 2014.

[175] Z. Zhang, S. Chen, H. Mei et al., "Ginkgo biloba leaf extract induces DNA damage by inhibiting topoisomerase II activity in human hepatic cells," Scientific Reports, vol. 5, p. 14633, 2015.

[176] J. W. Kang, J. H. Kim, and K. Song, "Kaempferol and quercetin, components of Ginkgo biloba extract (EGb 761), induce caspase-3-dependent apoptosis in oral cavity cancer cells," Phyther. Res.vol. 24, no. 1, 2010.

[177] A. J. Thompson, I. McGonigle, R. Duke, G. A. Johnston, and S. C. Lummis, "A single amino acid determines the toxicity of Ginkgo biloba extracts," The FASEB Journal: Official Publication of the Federation of American Societies for Experimental Biology, vol. 26, pp. 1884-91, 2012.

[178] Z. Cai, C. Wang, P. Liu, P. Shen, Y. Han, and N. Liu, "Ginkgo biloba extract in combination with sorafenib is clinically safe and tolerable in advanced hepatocellular carcinoma patients," Phytomedicine: International Journal of Phytotherapy and Phytopharmacology, vol. 23, pp. 1295-1300, 2016.

[179] M. S. Tan, J. T. Yu, C. C. Tan et al., "Efficacy and adverse effects of Ginkgo Biloba for cognitive impairment and dementia: a systematic review and meta-analysis," Journal of Alzheimer's Disease: JAD, vol. 43, pp. 589-603, 2015.

[180] T. A. Aziz, S. A. Hussain, T. O. Mahwi, Z. A Ahmed, H. S Rahman, and A Rasedee, "The efficacy and safety of Ginkgo biloba extract as an adjuvant in type 2 diabetes mellitus patients ineffectively managed with metformin: a double-blind, randomized, placebo-controlled trial," Drug Design, Development and Therapy, vol. 12, pp. 735-742, 2018.

[181] Y. Fan, X. Jin, C. Man, and D. Gong, "Does adjuvant treatment with ginkgo biloba to statins have additional benefits in patients with dyslipidemia?" Frontiers in Pharmacology, vol. 60, 2018.
[182] C. Schwarzbach, C. Vauth, S. Roll, S. N. Willich, and S. Weinmann, "Effects of Ginkgo biloba in dementia: systematic review and meta-analysis," BMC Geriatrics, vol. 10, no. 1, p. 14, 2010.

[183] P. L. Le Bars, M. Kieser, and K. Z. Itil, “A 26-week analysis of a double-blind, placebo-controlled trial of the ginkgo biloba extract EGb 761 in dementia," Dementia and Geriatric Cognitive Disorders, vol. 11, no. 4, pp. 230-237, 2000.

[184] S. Kanowski and R. Hoerr, "Ginkgo biloba extract EGb $761^{\circledR}$ in dementia: intent-to-treat analyses of a 24 -week, multicenter, double-blind, placebo-controlled, randomized trial," Pharmacopsychiatry, vol. 36, no. 6, pp. 297-303, 2003.

[185] L. Schneider, S. DeKosky, M. Farlow, P. Tariot, R. Hoerr, and M. Kieser, "A randomized, double-blind, placebo-controlled trial of two doses of ginkgo biloba extract in dementia of the alzheimers type," Current Alzheimer Research, vol. 2, no. 5, pp. 541-551, 2005.

[186] R. Ihl, N. Bachinskaya, A. D. Korczyn, and V. Vakhapova, "Efficacy and safety of a once-daily formulation of Ginkgo biloba extract EGb 761 in dementia with neuropsychiatric features: a randomized controlled trial," International Journal of Geriatric Psychiatry, vol. 26, no. 11, pp. 1186-1194, 2011.

[187] H. Herrschaft, A. Nacu, S. Likhachev, I. Sholomov, R. Hoerr, and S. Schlaefke, "Ginkgo biloba extract EGb 761 in dementia with neuropsychiatric features: a randomised, placebocontrolled trial to confirm the efficacy and safety of a daily dose of $240 \mathrm{mg}$," Journal of Psychiatric Research, vol. 46, no. 6, pp. 716-723, 2012.

[188] M. Rapp, M. Burkart, T. Kohlmann, and J. Bohlken, "Similar treatment outcomes with Ginkgo biloba extract EGb 761 and donepezil in Alzheimer's dementia in very old age: a retrospective observational study," International Journal of Clinical Pharmacology \& Therapeutics, vol. 56, no. 3, pp. 130-133, 2018.

[189] O. Szczurko, N. Shear, A. Taddio, and H. Boon, "Ginkgo biloba for the treatment of vitilgo vulgaris: an open label pilot clinical trial," BMC Complementary and Alternative Medicine, vol. 11, p. 21, 2011.

[190] B. Salehi, R. Imani, M. R. Mohammadi et al., "Ginkgo biloba for Attention-Deficit/Hyperactivity Disorder in children and adolescents: a double blind, randomized controlled trial," Progress in Neuro-Psychopharmacology and Biological Psychiatry, vol. 34, no. 1, pp. 76-80, 2010. 